\title{
Moneta e cultura giuridica
}

\section{Un brocardo di Azzone nella costruzione del diritto pecuniario medievale}

David De Concilio

\begin{tabular}{|l|l|}
\hline Date of deposit & 19122019 \\
\hline Document version & Author's accepted manuscript \\
\hline Access rights & $\begin{array}{l}\text { C Koninklijke Brill NV, Leiden, 2019. This work is made available } \\
\text { online in accordance with the publisher's policies. This is the } \\
\text { author created, accepted version manuscript following peer } \\
\text { review and may differ slightly from the final published version. }\end{array}$ \\
\hline $\begin{array}{l}\text { Citation for } \\
\text { published version }\end{array}$ & $\begin{array}{l}\text { De Concilio, D. (2019). Moneta e cultura giuridica: Un brocardo di } \\
\text { Azzone nella costruzione del diritto pecuniario medievale. }\end{array}$ \\
\hline $\begin{array}{l}\text { Link to published } \\
\text { version }\end{array}$ & \begin{tabular}{l} 
https://doi.org/10.1163/15718190-00870A07 \\
\hline
\end{tabular}
\end{tabular}

Full metadata for this item is available in St Andrews Research

Repository at: https://research-repository.st-andrews.ac.uk/

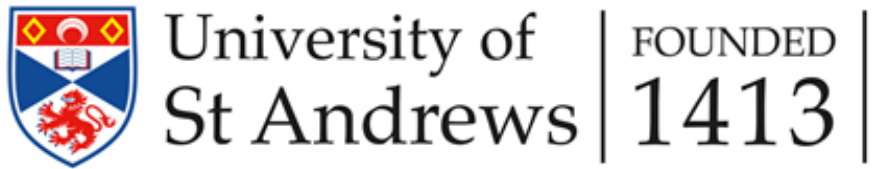




\section{Moneta e cultura giuridica.}

Un brocardo di Azzone nella costruzione del diritto pecuniario medievale

Da tempo la storiografia ha riconosciuto come un fatto consolidato l'importanza che il Medioevo - e in particolare le grandi trasformazioni del XII e XIII secolo - ebbe nello sviluppo del pensiero economico occidentale, sulla scia della cosiddetta rivoluzione commerciale. L'attenzione degli studiosi non poteva che cadere su uno degli aspetti fondamentali di questo fenomeno, ossia sulla dimensione centrale che il tema monetario assunse proprio in quei secoli, sulla sua teorizzazione e sulla sua sistemazione intellettuale. Gli studi in questo senso si collocano crocevia della multidisciplinarietà: dalla storia economica e monetaria stricto sensu ${ }^{1}$, fino a quella del pensiero filosofico e teologico ${ }^{2}$.

Anche la storiografia giuridica non è stata indifferente al tema economico e monetario, e in particolare ha indagato il concetto di moneta e i problemi giuridici da essa derivanti nell'elaborazione dottrinaria del diritto colto medievale, per quanto attiene sia all'età della Glossa che a quella del Commento $^{3}$. Come infatti si vedrà in maggiore dettaglio più avanti, la cultura giuridica, fin dalla

\footnotetext{
${ }^{1}$ Per quanto riguarda la storia economica, tutt'ora fondamentali rimangono gli studi classici di Marc Bloch, Carlo Cipolla e Roberto S. Lopez. Si vedano in particolare: M. Bloch, Esquisse d'une histoire monétaire de l'Europe, Paris 1954; C. M. Cipolla, Moneta e civiltà mediterranea, Venezia 1957 e Id., Le avventure della lira, Milano 1958; R. S. Lopez, The commercial revolution of the middle ages, 950-1350, Englewood Cliffs 1971. In tempi più recenti si segnalano le opere di P. Spufford, Money and its use in Medieval Europe, Cambridge 1988 e T. J. Sargent [e] F. R. Velde, The big problem of small change, Princeton, Oxford 2002.

${ }^{2}$ Questo aspetto è stato esaminato in modo esteso dagli studiosi, in ragione della sua primaria importanza nell'evoluzione del pensiero economico. Si segnalano in particolare: O. Bazzichi, Dall'usura al giusto profitto. L'etica economica della Scuola francescana, Cantalupa 2008; P. Evangelisti, Il pensiero economico nel Medioevo. Ricchezza, povertà, mercato e moneta, Roma 2016; O. Langholm, Economics in the Medieval schools. Wealth, exchange, value, money and usury according to the Paris theological tradition: 1200-1350, Leiden 1992; G. Todeschini, I mercanti e il tempio. La società cristiana e il circolo virtuoso della ricchezza fra Medioevo ed età moderna, Bologna 2002.

3 Tutt'ora fondamentale sull'argomento rimane l'opera di W. Taeuber, Geld und Kredit im Mittelater, Berlin 1933. Si segnalano anche G. Salvioli, Moneta (Diritto monetario), in: Enciclopedia Giuridica Italiana, 10, parte 3, Milano 1901, p. 16-128 e P. Grossi, Ricerche sulle obbligazioni pecuniarie nel diritto comune, Milano 1960. In epoca più recente sono da menzionare gli studi di W. Ernst, The Glossators' monetary law, in: The Creation of the Ius Commune: From Casus to Regula, a cura di J. W. Cairns [e] P. J. Du Plessis, Edinburgh 2010, p. 219-246; Id., Mensura et mensuratum: money as measure and measure for money, in: Money as God? The monetization of the Market and its impact on religion,
} 
propria rinascita nella culla bolognese, fu sensibile ai problemi monetari del proprio tempo, e nello specifico alle esigenze di un mercato che proprio in quegli anni e in quella medesima area geografica vedeva una rapida ripresa ed espansione. Basti per ora accennare che i glossatori civilisti, secondo la metodologia che è loro propria, guardarono alla moneta come istituzione giuridica attraverso i testi del Corpus iuris: le prime testimonianze della loro elaborazione sul tema sono contenute nelle opere dei giuristi Bulgaro (XII sec) ${ }^{4}$ e Pillio da Medicina (m. dopo il 1207) ${ }^{5}$, per trovare infine una compiuta sistemazione nel testo che ci accingiamo a esaminare in questa sede: un passo di quella raccolta di Brocarda di Azzone che la storiografia ha ribattezzato 'Salicta', il quale rappresenta un punto di svolta in questo percorso dottrinale.

Ciò premesso, bisogna chiedersi quale sia l'opportunità - e in un certo senso la necessità - di rinnovare la ricerca sull'elaborazione del concetto giuridico di moneta nei glossatori, e in particolare su di un passo, quale appunto il suddetto brocardum di Azzone, già ampiamente esaminato dalla storiografia $^{7}$. Le ragioni a mio avviso sono due: una attinente allo stato attuale della ricerca e un'altra alle sue conclusioni.

Per quanto riguarda il primo aspetto, sono necessarie alcune puntualizzazioni rispetto allo studio dei Brocarda, sia in generale, sia nello specifico di quello di nostro interesse. Dacché Emil Seckel, più di un secolo fa, sottolineò la necessità di un'indagine approfondita della tradizione autografa dei Brocarda di Azzone ${ }^{8}$, non è stato intrapreso ancora alcuno studio critico sul testo di portata generale. Se prima l'opera e la sua genesi erano avvolte da una certa oscurità, certamente gli

politics, law, and ethics, a cura di J. von Hagen [e] M. Welker, Cambridge 2016, p. 60-79; si v. infine l'opera collettiva Money in the Western legal tradition: Middle Ages to Bretton Woods, a cura di D. Fox [e] W. Ernst, New York 2016.

${ }^{4}$ Per approfondire v. B. Paradisi, Bulgaro, in: Dizionario Biografico degli Italiani (DBI), 15, Roma 1972, ad vocem; L. Loschiavo, Bulgaro, in: Dizionario Biografico dei Giuristi Italiani (DBGI), 1, Bologna 2013, p. 357-359.

${ }^{5}$ Per approfondire v. E. Conte, Pillio da Medicina, in: DBI (supra, n. 4), 83, Roma 2015, ad vocem; E. Cortese, Pillio, in: DBGI (supra, n. 4), 2, Bologna 2013, p. 1587-1590.

${ }^{6}$ v. P. Legendre, La pénétration du droit romain dans le droit canonique classique de Gratien à Innocent IV (1140-1254), Paris 1964, p. 80; P. Weimar, Die legistische Literatur der Glossatorenzeit, in: Handbuch der Quellen und Literatur der neueren europäischen Privatrechtsgeschichte, Erster Band: Mittelalter (1100-1500), die gelehrten Rechte und die Gesetzgebung, a cura di Helmut Coing, München 1973, p. 129-260, p. 239 n. 5; M. Schwaibold, Wer sucht, der findet, Rechtshistorisches Journal 4 (1985), p. 202-214, p. 214 n. 49.

${ }^{7}$ Cfr. Taeuber, op. cit. (supra, n. 3), p. 228-231, 328-330; Ernst, The Glossators' (supra, n. 3), p. 234-236; Sargent, Velde, op. cit. (supra, n. 1), p. 75-77.

${ }^{8}$ E. Seckel, Distinctiones glossatorum. Studien zur Distinktionen-Literatur der romanistischen Glossatorenschule, verbunden mit Mitteilungen unedierter Texte, in: Festschrift der Berliner juristischen Fakultät für Ferdinand von Martitz zum Fünfzigjährigen Doktorjubiläum am 24. Juli 1911, Berlin 1911 [rist. anast. Graz 1956], p. 384 n. 2. 
studi di Weimar hanno permesso di chiarire numerosi punti ${ }^{9}$; ciò nonostante, tuttavia, si è ancora lontani da un lavoro esaustivo sul testo, né ne esiste ancora una edizione moderna che sia universalmente accessibile.

Questa incompletezza della nostra conoscenza dell'opera nel suo complesso si ripercuote naturalmente anche sugli studi del brocardo di Azzone in tema di moneta: tutti gli autori che hanno fronteggiato la materia sono stati fortemente tributari del lavoro di Taeuber, l'unico finora a essersi cimentato nella ricostruzione testuale di questo brocardo ${ }^{10}$. Eppure, proprio tale ricostruzione difetta dell'accuratezza necessaria: lo studioso tedesco utilizzò a tal fine una sola delle quattro recensioni a stampa dell'opera - quella prodotta a Basilea da Caspar Hervag nel 1567, che risente come tutte le altre di forti rimaneggiamenti testuali da parte dell'editore - e la confrontò con un solo manoscritto (ms. Bamberg, StaatsB Jur. 34), a fronte degli almeno trentasei in cui il passo è presente. Oltre evidentemente a non consentire una ricostruzione testuale soddisfacente del brocardo, ciò induce Taeuber a fraintendere o a non riuscire ad individuare alcune delle allegazioni del Corpus iuris contenute nel passo. Così ad esempio al $\S 40$ non riuscì a sciogliere l'allegazione di D. 35.1.28 ('sicut et in ff. de conditionibus et demonstrationibus, Filie $\S$ ultimo'), poiché l'edizione di Caspar Hervag riportava erroneamente 'sic Institutionibus, de conditione indebiti, l. filiae $\S$ ultimo' ${ }^{11}$. $\mathrm{O}$ ancora, intese l'allegazione del $\S 9$ come D. 32.93 ('D. de legatis 3 l. Lucius Titius'), invece del più pertinente D. 31.1.22, a causa della mancata collazione di manoscritti contenenti la variante corretta ('ff. de legatis II Lucius Titius') $)^{12}$.

Per questa ragione ho ritenuto necessario esaminare l'elaborazione dei glossatori in tema monetario - e nello specifico il brocardo di Azzone che ne rappresenta la compiuta formulazione partendo dalla ricostruzione della tradizione manoscritta, nella convinzione che l'esercizio dell'edizione critica rappresenti il miglior modo di entrare in contatto con il tema sostanziale che ci interessa.

Una edizione critica integrale del testo dei Brocarda di Azzone sarebbe opera auspicabile, ma assai gravosa. Perciò ho ritenuto opportuno procedere almeno a un'edizione in riferimento al particolare testo di nostro interesse per la storia del diritto in tema di moneta, quel brocardo 'eadem mensura vel moneta debetur' di cui si è detto. Un solo passaggio dell'ampia raccolta azzoniana, la cui tradizione manoscritta può risultare però meglio comprensibile grazie al necessario lavoro di

\footnotetext{
${ }_{9}^{9}$ P. Weimar, Argumenta brocardica, Studia Gratiana 14 (1967) [Collectanea Stephan Kuttner, 4], p. 91-123.

${ }^{10}$ v. Taeuber, op. cit. (supra, n. 3), p. 328-330.

${ }^{11}$ Ivi, p. 328.

${ }^{12}$ Ivi, p. 329.
} 
recensione dei testimoni ${ }^{13}$. Come ogni altra costruzione della dottrina giuridica dell'età della glossa, anche il tema della moneta risalta grazie alla pratica sistematica del metodo dialettico, che è particolarmente evidente nel genere dei brocarda: un genere assai poco attraente per il lettore moderno e perciò un po' trascurato dalla storiografia, ma che merita senza dubbio maggiore attenzione.

La seconda ragione alla base del presente studio, come accennato, riguarda la necessità di rivalutare l'importanza dell'apporto fornito dai glossatori nell'elaborazione di un principio giuridico in tema pecuniario: un contributo che la storiografia, giuridica e non, ha teso forse eccessivamente a sottovalutare, come si avrà modo di dire nel corso del presente lavoro. Per mettere in evidenza l'opportunità di partire dal nostro brocardo e dalla sua struttura dialettica, nelle pagine successive ci si concentrerà in particolare su: I) le premesse storiche e giuridiche necessarie alla comprensione del contesto di nascita del brocardo; II) il genere dei Brocarda; III) il testo, la sua tradizione e la sua circolazione; IV) il contenuto e la portata dottrinaria del brocardo di Azzone; V) l'edizione che qui si intende presentare.

I.

Premesse storico-giuridiche

\section{Il contesto monetario}

Pur non essendo lo scopo di questo lavoro tracciare un quadro dettagliato ed esaustivo del sistema monetario nell'età dei glossatori, sono tuttavia necessari alcuni brevi accenni, al fine di poter comprendere le questioni e le prospettive della scienza giuridica a tal proposito. Al tempo dei primi glossatori e fino a poco dopo l'età di Azzone (1150 ca-1230 ca) ${ }^{14}$, l'Occidente latino utilizzava un sistema monetario basato su un unico metallo, l'argento, secondo un modello ereditato dall'impero carolingio $^{15}$. Alla sua origine, tale sistema si basava su un'unica denominazione, il cd. denaro d'argento, che risultava pertanto difficoltoso e sconveniente sia per le transazioni al minuto - le quali

\footnotetext{
${ }^{13}$ v. infra, parte III $\S 5$.

${ }^{14}$ Per approfondire v. P. Fiorelli, Azzone, in: DBI (supra, n. 4), 4, Roma 1962, ad vocem; E. Conte, L. Loschiavo, Azzone, in: DBGI (supra, n. 4), 1, Bologna 2013, p. 137-139.

${ }^{15}$ Sull'argomento v. Bloch, op. cit. (supra, n. 1), p. 31.
} 
richiedevano quantità di metallo prezioso di molto inferiori a quella contenuta in un singolo denaro - sia per quelle su larga scala, dove si necessitava di computare grandi quantità di denari. Per ovviare a tale inconveniente la moneta reale coniata era affiancata da una serie di unità monetarie puramente di conto, che fungevano da multipli del denaro: così 12 denari componevano un solido e 20 solidi una $\operatorname{lira}^{16}$.

La dissoluzione dell'impero carolingio causò una serie di dissesti monetari (che portarono a una progressiva svalutazione del denaro, e di conseguenza a slittamenti nell'equivalenza tra la moneta reale e quella di conto) e la frammentazione delle autorità politiche autorizzate a battere moneta. Da ciò derivò anche la frammentazione dello standard monetario: i vari denari locali, pur mantenendo sotto varie espressioni la stessa denominazione, iniziano a differenziarsi tra loro per peso e qualità. Ciò comportò la differenziazione non solo tra le varie monete effettive, ma anche tra quelle immaginarie, con ogni lira diversa dalle altre ${ }^{17}$.

Il quadro, già estremamente frammentario, agli albori del XIII secolo si complicò ulteriormente. Proprio negli anni a cavallo della redazione dei Brocarda 'Salicta'18, infatti, le città mercantili italiane - in testa Venezia e Genova - iniziarono ad emettere un nuovo tipo di moneta di maggior valore che affiancò l'ormai svalutato denaro: il cosiddetto (denaro) grosso o ducato d'argento ${ }^{19}$. Come risultato di questo ambiente monetario - fortemente eterogeneo e soggetto a costanti fluttuazioni nel rapporto tra le varie monete, in conseguenza di una perenne inflazione inframezzata da interventi deflattivi - si rese sempre più necessario e strategico il ricorso a un sistema di cambi che permettesse di scambiare tra di loro le diverse valute, calcolando l'equivalenza tra una data quantità di monete di un certo tipo e il suo corrispettivo in monete di un tipo diverso, ossia il ricorso una unità di misura comune: la moneta di conto.

\footnotetext{
${ }^{16}$ Cipolla, Le avventure (supra, n. 1), p. 20, 22 n. 9; Id., Moneta (supra, n. 1), p. 53; Ernst, Mensura (supra, n. 3), p. 6162, 66; Salvioli, op. cit. (supra, n. 3), p. 40.

${ }^{17}$ Cipolla, Le avventure (supra, n. 1), p. 23-26, 30; Ernst, The Glossators' (supra, n. 3), p. 223; Salvioli, op. cit. (supra, n. 3), p. 42, p. 53-59; Sargent, Velde, op. cit. (supra, n. 1), p. 45; Spufford, op. cit. (supra, n. 1), p. 100, $101,104$.

${ }^{18}$ Weimar, Argumenta (supra, n. 9), p. 121, dimostra come la stesura del lavoro di Azzone non possa essere precedente al 1208-1210, poiché egli cita la Summa Codicis, che dovrebbe essere stata scritta in quegli anni. F. Patetta, I libri legali e il corredo d'un giudice bolognese nell'anno 1211, e un caso di rappresaglia fra Bologna e Ferrara, Atti della Reale Accademia delle scienze di Torino, 50 (1914-15), p. 1174, riporta in ogni caso l'esistenza dell'opera già nel 1211. La diffusione dei primi grossi avviene tra il 1201 e il 1222.

${ }^{19}$ Sull'argomento v. Spufford, op. cit. (supra, n. 1), p. 98-99, 225-228; Sargent, Velde, op. cit. (supra, n. 1), p. 65, 160; Cipolla, Le avventure (supra, n. 1), p. 44-45, 49.
} 
Questa situazione favorì un maggiore intervento del potere politico sulla moneta: infatti, dovendo rispondere all'esigenza di permettere commerci e scambi basati sull'interazione di diverse monete locali, si rese necessaria, da parte dell'autorità pubblica, la fissazione di un valore nominale che superasse le differenze sostanziali tra le monete. Questo fenomeno potrebbe leggersi parallelamente alla crescita di importanza del potere politico nel diritto penale, che venne sottratto alla negoziazione fra parte lesa e offensore e che condusse - proprio come ora si vedrà che avvenne in ambito monetario - a usi arbitrari di tale potere d'intervento ${ }^{20}$. Si aprì così facilmente la strada al fenomeno delle mutazioni monetarie, ossia all'alterazione del valore della moneta da parte del sovrano. Ciò poteva avvenire sia alterando materialmente il contenuto metallico intrinseco di una denominazione monetaria, sia semplicemente variando il rapporto di equivalenza tra monete diverse. La possibilità di ricorrere a tali mutazioni nominali era resa possibile dall'assenza sulle monete reali di qualsivoglia indicazione non solo del peso e della finezza, ma anche del valore, che era dunque determinato esclusivamente in virtù delle tariffe fissate dagli statuta. I sovrani e le autorità monetarie del Medioevo furono pienamente consapevoli di questa potenzialità e non lesinarono il ricorso alle mutazioni monetarie, che tra il XIII e il XIV secolo assunse i caratteri di un vero e proprio abuso ${ }^{21}$.

${ }^{20}$ Sulla costruzione dell'azione pubblica in ambito penale nelle città italiane, a partire dal XIII secolo, si vedano soprattutto gli studi di Mario Sbriccoli: M. Sbriccoli, Giustizia negoziata, giustizia egemonica. Riflessioni su una nuova fase degli studi di storia della giustizia criminale, in: Criminalità e giustizia in Germania e Italia. Pratiche giudiziarie e linguaggi giuridici tra tardo medioevo ed età moderna, a cura di M. Bellabarba, G. Schwerhoff, A. Zorzi, Bologna 2001, p. 345-364, p. 351, 360ss; Id., «Vidi communiter observari». L'emersione di un ordine penale pubblico nelle città italiane del secolo XIII, Quaderni fiorentini per la storia del pensiero giuridico moderno, 27 (1998), p. 231-268; Id., Legislation, justice and political power in Italian cities, 1200-1400, in: Legislation and Justice, a cura di A. Padoa-Schioppa, Oxford 1997, p. 37-55.

${ }^{21}$ Su quanto detto v. Bloch, op. cit. (supra, n. 1), p. 53-60; Ernst, The Glossators' (supra, n. 3), p. 224.

All'alterazione del valore della moneta da parte del potere pubblico faceva sovente da contraltare il tentativo del mercato di resistere alle mutazioni monetarie: non sempre, infatti, il corso legale della moneta (il valor impositus, fissato dalle tariffe) corrispondeva alla prassi mercantile, la quale tendeva a creare un proprio corso, diverso da quello ufficiale e rispondente alle esigenze del mercato (il valor usualis o currens). Questo fenomeno rendeva poi necessari ulteriori interventi da parte delle autorità pubbliche, al fine di adeguare il valore legale a quello corrente: v. Bloch, op. cit. (supra, n. 1), p. 49; Ernst. The Legists' Doctrines on Money and the Law, in: Money in the Western Legal Tradition (supra, $\mathrm{n}$. 3), p. 110-135, p. 126. Da un punto di vista giuridico, lo strumento che riconobbe l'operatività dei limiti che le prassi mercantili ponevano agli interventi pubblici in materia monetaria fu il ruolo della consuetudine (intesa come consuetudo loci), a cui i giuristi attribuirono la capacità di derogare al diritto positivo anche in questo campo, assegnando di fatto una valenza normativa alle esigenze del mercato. Basti qui accennare come ciò sia evidente sia in ambito canonistico (ad esempio nella decretale cd. 'Si beneficiorum', Clem. 3.8.2), così come tra i civilisti: v. su tutti Grossi, op. cit. (supra, $\mathrm{n}$. 3), p. 113-123, 378-380. 
Le mutazioni monetarie, largamente praticate, erano naturalmente viste con sfavore da parte dei gruppi di interesse della società medievale. Una pratica così odiosa per l'opinione pubblica coeva e che suscitava innumerevoli problemi e interrogativi, non poteva che ricevere attenzione da parte della classe intellettuale. I primi giuristi medievali manifestano perciò un certo interessamento per i temi e i problemi giuridici suscitati dalla situazione monetaria. Mentre assumono rapidamente un ruolo di riferimento per la cultura del loro tempo, essi formulano già una prima risposta alle esigenze della società medievale in cambiamento, iniziando la creazione di una dottrina per l'interpretazione giuridica della moneta, sulla base dell'adattamento delle categorie giuridiche mutuate dal diritto romano.

\section{L’elaborazione dei primi glossatori}

La pratica della mutazione monetaria, come si è visto, creava fenomeni speculativi e contrasti sociali, e poteva non di rado danneggiare gli interessi tanto delle classi dominanti - signori feudali e clero - quanto della nuova classe sociale emergente e in rapida espansione: il ceto mercantile. Attraverso la mutatio monetae prendevano corpo quindi una serie di problemi di carattere pratico, relativi specialmente alle obbligazioni pecuniarie. Chiamati a rispondere a tali problemi, i primi giuristi colti medievali partono dalla concezione della moneta come una merce: ogni moneta veniva valutata in base ai propri elementi costitutivi, ossia al contenuto intrinseco che essa aveva ${ }^{22}$.

La concezione della moneta-merce esplicava i propri effetti in particolare rispetto alle conseguenze di una mutazione monetaria sui debiti pecuniari. Il primo problema che i civilisti posero fu quello del momento da prendere a riferimento per la determinazione del valore della somma pecuniaria da rendere (ossia dell'unità di misura) e della qualità delle stesse monete, operando una scelta tra il tempus dationis e il tempus solutionis. In modo più semplice, che cosa accade, ad esempio, se l'unità di conto con cui è stato calcolato il debito cambia nel corso del tempo?

La prima formulazione a noi nota di tale quesito non riguarda di per sé la mutatio monetae, ma in generale la variazione delle unità di misura nel tempo, ed esercitò grande influenza sulla dottrina monetaria successiva. Essa ci è pervenuta attraverso una quaestio del giurista Bulgaro ${ }^{23}$ sulla

\footnotetext{
${ }^{22}$ Sargent, Velde, op. cit. (supra, n. 1), p. 70; Ernst, The Glossators' (supra, n. 3), p. 225-228.

${ }^{23} \mathrm{Si}$ tratta di una quaestio contenuta nella cosiddetta Collectio Parisiensis, ms. Paris, Bibliothèque nationale de France, lat. 4603, pubblicata in Quaestiones Dominorum Bononiensium, a cura di G. B. Palmieri, in BIMAE [Scripta Anecdota Glossatorum], 1, a cura di A. Gaudenzi, Bologna 1914², LX. Essa corrisponde alla quaestio LXIII dell'altra grande collezione di questioni di Bulgaro, la Collectio Gratianopolitana, contenuta nel ms. Grenoble, Bibliothèque municipale,
} 
mutazione dell'unità di misura degli aridi ${ }^{24}$ utilizzata a Bologna, lo staio; la quaestio di Bulgaro è ripresa poi da Pillio da Medicina in due sue quaestiones. La prima, a noi giunta come quaestio 79 della sua raccolta di Quaestiones sabbatinae ${ }^{25}$, consiste in uno sviluppo del caso già affrontato da Bulgaro. Con la seconda quaestio invece, risalente probabilmente al $1182^{26}$ e nota come Quidam Creditor Lucenses ${ }^{27}$, Pillio estende il caso dello staio bolognese alle obbligazioni pecuniarie, trattando l'eventualità di sopravvenuta svalutazione della moneta.

Pur non potendo procedere in questa sede a esaminare le tre quaestiones nel dettaglio, basti qui rilevare come tutte e tre sono accomunati dalla medesima fattispecie di base: è stata contratta un'obbligazione secondo una determinata unità di misura e successivamente tale unità ha subito un mutamento, nello specifico una riduzione. Se quindi il debitore si liberasse pagando attenendosi al valore nominale della vecchia unità, realizzerebbe un guadagno e il creditore incorrerebbe in una perdita, pagando un valore reale delle monete inferiore a quanto inizialmente pattuito. Se invece egli pagasse con riguardo al contenuto intrinseco inizialmente pattuito, indipendentemente dall'unità di misura, il creditore sarebbe tutelato. Ed è questa interpretazione che i giuristi seguono nelle tre quaestiones.

Il fatto che sia Bulgaro che Pillio propendano per delle soluzioni orientate alla tutela delle parti contraenti a dispetto degli interessi del potere pubblico, non deve tuttavia far ritenere che al tempo dei primi glossatori la materia fosse affatto pacifica in dottrina. Anzi, vi sono tutte le ragioni per pensare che sia le tre quaestiones sopra menzionate, sia il brocardo di Azzone che ci accingiamo

391.1, pubblicata sempre ivi. Sulle due edizioni v. A. Belloni, Le questioni civilistiche del secolo XII. Da Bulgaro a Pillio da Medicina e Azzone [Ius commune Sonderhefte. Studien zur Europäischen Rechtsgeschichte, 43], Frankfurt am Main 1989, p. 7-13. Sull'argomento v. Ernst, The Glossators' (supra, n. 3), p. 229-231, e Sargent, Velde, op. cit. (supra, n. 1), p. 77; Taeuber, op. cit. (supra, n. 3), p. 219-222.

${ }^{24}$ Vale a dire le 'sostanze solide incoerenti, misurabili secondo misure di capacità, in modo analogo ai liquidi (grano, sabbia, ecc.)': v. àrido, in: Vocabolario Treccani online, www.treccani.it/vocabolario, consultato il 19/09/2018, ad vocem; cfr. anche ivi, s.v. stàio.

${ }^{25}$ Pillio, Quaestiones Sabbatinae, Roma 1560 [rist. anast. in Corpus Glossatorum Iuris Civilis, 4, a cura del Centro di studi di storia del diritto italiano dell'Università degli Studi di Torino, Torino 1973], quaestio 79, p. 145-147 (Belloni, op. cit. (supra, n. 23), p. 109); v. anche Grossi, op. cit. (supra, n. 3), p. 95-97.

${ }^{26}$ Taeuber, op. cit. (supra, n. 3), p. 119, 121 e 122, che riporta l'indiscussa opinione di Savigny (v. F. C. Savigny, Geschichte des römischen Rechts im Mittelalter, 4, Darmstadt 1956, p. 319-320).

${ }^{27}$ Contenuta anch'essa nelle Quaestiones sabbatinae. In Belloni, op. cit. (supra, n. 23), p. 102 l'incipit è 'Quidam creditor Bononiensis a quinquennio retro Lucenses amico suo numeravit'. La più antica versione a stampa è in Pillio, Celeberrimi iure cons. ac glosatoris vetustissimi. D. Pilei Modicensis Quaestiones aureae, a cura di A. Blado, Roma 1560, p. 62-64. v. Taeuber, op. cit. (supra, n. 3), p. 119-126; Ernst, The Glossators' (supra, n. 3), p. 231-234. 
a esaminare, pur rappresentando le prime testimonianze a noi pervenute della questione, siano con tutta probabilità solo il punto d'arrivo di una discussione tra i legisti, che vide certamente anche prese di posizione controcorrente, nel senso di una prevalenza degli statuta sul diritto privato ${ }^{28}$.

È a seguito di queste prime attestazioni dottrinarie e in stretta connessione con esse che si colloca l'opera che ci accingiamo a esaminare, un passo attribuito ad Azzone e contenuto, come si è detto, nei suoi Brocarda. In questo testo, come si vedrà, il giurista bolognese affronta il problema della variazione delle unità di misura e delle monete nel tempo, accogliendo le posizioni di fondo dei suoi predecessori e sancendo un principio generale che avrà enorme successo tra le generazioni di giuristi successive. Tuttavia, prima di passare all'esame del contenuto del brocardo, bisogna soffermarsi sulla storia dell'opera e del genere letterario a cui appartiene.

II.

I Brocarda

${ }^{28}$ v. Taeuber, op. cit. (supra, n. 3), p. 225. Il metodo dei glossatori - che tendevano a tramandare l'opinione prevalente non ha lasciato molte tracce di tale dibattito, tuttavia se ne ritrova un'eco, in ambiente canonistico, in una glossa anonima del 1200 al canone Quis ignoret del Decretum Gratiani (C. 32 q. 4 c. 6), contenuta nel Ms. Bamberg, StaatsBibliothek, can. 13 , fol. $207 \mathrm{v}$ : il testo glossato contiene il principio canonistico 'serviamus et nos legi et tempori nostro', ossia la necessità che ogni tempo storico abbia la sua legge, secondo il volere della Provvidenza. L'autore della glossa applica questo principio al caso di obbligazioni pecuniarie a seguito di mutazione monetaria, sostenendo un'interpretazione "statalista" e favorevole alle istanze del potere pubblico: egli vieta dunque l'utilizzo di alcuna misura o moneta precedente e in contrasto con la legge vigente: Taeuber, op. cit. (supra, n. 3), p. 226 e ivi n. 646; T. Bisson, Conservation of coinage. Monetary exploitation and its restraint in France, Catalonia, and Aragon (c.A.D.1000-c.1225), Oxford 1979, p. 179-180. Lungi dall'essere un episodio sporadico, la glossa in questione viene ripresa da Giovanni Teutonico (m. 1245) e da Vincenzo Ispano (m. 1248), a testimonianza di un certo dibattito dottrinario e dell'esistenza di una posizione nominalistica minoritaria: v. glossa a 3 Comp 3.37.5, 'vel estimationem', in: Ms Admont, Stiftsbibliothek, 22, citato da ERNST, The Glossators' (supra, n. 3), p. 242 n. 82, e riportato da K. Pennington, Medieval Legal History, legalhistorysources.com/edit323.htm, consultato il 06/01/2018; v. anche Bisson, Conservation (supra, n. 28 ), p. 179.

Seppure le tracce di posizioni minoritarie al riguardo nel diritto colto sono sporadiche e sembrano sparire presto, l'impressione che l'affermazione del metallismo e della tutela dei contraenti sia stato il risultato di un processo dialettico emerge anche dalla testimonianza di Cino da Pistoia (1270-1336): v. Cino da Pistoia, In Codicem, et aliquot titulos primi Pandectarum tomi, id est, Digesti veteris, doctissima commentaria, Frankfurt am Main, 1578, fol. 50v. Sul passo di Cino v. Grossi, op. cit. (supra, n. 3), p. 358-359, 


\section{I brocarda in generale}

Prima di passare all'analisi dei Brocarda azzoniani e del nostro brocardo nello specifico, è necessaria - senza alcuna pretesa di esaustività e rinviando ai maggiori studi a tal proposito ${ }^{29}$ - una breve disamina del genere letterario, e in primis sulla sua nascita. Il termine Brocarda è stato utilizzato per la prima volta da Pillio da Medicina, ma non per il suo Libellus disputatorius, che contiene quelli che noi oggi chiamiamo appunto brocardi, bensì per le sue Quaestiones, che con la loro opposizione dialettica rappresentano un genere letterario affine ai brocarda $^{30}$. L'etimologia stessa del termine è incerta: già Savigny respinse l'ipotesi tradizionale per cui esso possa derivare dal nome di Burcardo di Worms ${ }^{31}$. Hermann Kantorowicz ipotizzò la sua derivazione da una deformazione di 'pro-contra' in 'procarda'32. Weimar ha successivamente teorizzato l'origine del termine dalla parola mediolatina 'broccus', ossia 'dai denti sporgenti' 33 .

In ogni caso, i brocarda trovano la loro origine - come quasi tutti i generi letterari dei glossatori - nelle glosse marginali dei manoscritti, e nello specifico nei notabilia, ossia glosse finalizzate all'esposizione di un concetto giuridico, identificabili con gli argumenta, ossia prove fondamentali secondo i principi della retorica ${ }^{34}$. Già nei notabilia stessi si trovano i contra che caratterizzano il genere brocardico ${ }^{35}$, mentre solo successivamente, con il Perpendiculum, il Libellus

\footnotetext{
${ }^{29}$ Weimar, Argumenta (supra, n. 9); S. Kuttner, Réflexions sur les brocards des glossateurs, in: Mélanges Joseph de Ghellnick II, Gembloux 1951, p. 767-792. In tempi più recenti, si è interessato al tema dei brocarda, dal differente punto di vista degli studi sull'argomentazione giuridica (seppure con frequenti incursioni nella storia del diritto), lo studioso D. V. Dalbrenta, Brocardica. Una introduzione allo studio e all'uso dei brocardi. Principi di filosofia forense, Milano 2007.

${ }^{30}$ Savigny, op. cit. (supra, n. 26), 3, p. 569 e Seckel, Ueber neuere Editionen juristischer Schriften aus dem Mittelalter.
} I, Zeitschrift der Savigny-Stiftung für Rechtsgeschichte: Romanistische Abteilung, 21 (1900), p. 289; v. anche Kuttner, Réflexions (supra, n. 29), p. 768 ed E. Genzmer, Die iustinianische Kodifikation und die Glossatoren, in: Atti del congresso internazionale di diritto romano (Bologna, 1933), 1, Pavia 1934, p. 426.

${ }^{31}$ Savigny, op. cit. (supra, n. 26), 3, p. 569.

${ }^{32}$ H. Kantorowicz, The quaestiones disputatae of the Glossators, Tijdschrift voor Rechtsgeschiedenis, 16 (1937-1938), p. 4. Kuttner, Réflexions (supra, n. 29), p. 768, sembra propendere per questa ipotesi, così come Legendre, op. cit. (supra, n. 6), p. 80-81.

${ }^{33}$ v. Weimar, Argumenta (supra, n. 9), p. 91, 105-109, sia per quanto riguarda la sua ipotesi che la storia delle ricostruzioni etimologiche. Per un excursus sull'origine del termine v. anche Dalbrenta, op. cit. (supra, n. 29), p. 21-22.

${ }^{34}$ Savigny, op. cit. (supra, n. 26), 3, p. 567; Weimar, Argumenta (supra, n. 9), p. 91; Kuttner, Réflexions (supra, n. 29), p. 770; Genzmer, op. cit. (supra, n. 30), p. 423; A. M. Stickler, Sacerdotium et Regnum nei decretisti e nei primi decretalisti. Considerazioni metodologiche di ricerca e testi, Salesianum 15 (1953), p. 5-42, p. 9-10. Sui notabilia v. anche S. Kuttner, Repertorium der Kanonistik [Studi e Testi 71], Città del Vaticano 1937, p. 232-233, e sui brocarda ivi, p. 239.

35 Stickler, op. cit. (supra, n. 34), p. 9; Kuttner, Réflexions (supra, n. 29), p. 777. 
Disputatorius di Pillio e i Brocarda attribuiti ad Ottone da Pavia, il brocardo si sviluppò come un genere a sé stante, oggetto di raccolte specifiche separate dalla pagina del testo normativo ${ }^{36}$.

Passando alla sua struttura, si può notare come il brocardo, nel suo compiuto sviluppo, sia tripartito: è infatti composto dall'enunciazione di una massima giuridica, dalla citazione di testi antitetici e da una soluzione che risolve le antinomie; questa impostazione dialettica non è rara nell'opera dei glossatori ed è comune anche ad altri generi letterari, come ad esempio le quaestiones. Gli studiosi si sono soffermati a esaminare la tripartizione del brocardo e in particolare a chiedersi quale ne sia l'elemento caratterizzante. Così ad esempio Genzmer pose l'accento sull'opposizione dialettica dei brocardi: il fatto che una massima sia seguita dal suo opposto dovrebbe servire a evitare il rischio di una rigida generalizzazione della regola giuridica ${ }^{37}$. Anche Kuttner ritenne che, almeno nella fase iniziale di sviluppo del brocardo, il contrarium fosse un suo elemento caratteristico, volto a verificarne l'attitudine a servire da regola generale: proprio la presenza di un'opposizione dialettica permetterebbe di distinguere tra un generale e un notabile o un argumentum ${ }^{38}$. Weimar, seguendo le considerazioni di Kuttner, ha individuato proprio nella duplicità il carattere essenziale del genere letterario ${ }^{39}$.

Per quanto riguarda invece le solutiones, si tratta di un elemento inizialmente non presente nel genere, aggiuntosi in una fase successiva per applicazione del metodo scolastico della solutio contrariorum: per questa ragione Kuttner non le considera un elemento essenziale della struttura del brocardo, pur costituendone la parte più interessante per la storia delle dottrine giuridiche ${ }^{40}$.

\footnotetext{
${ }^{36}$ Stickler, op. cit. (supra, n. 34), p. 10. Sulle prime due opere menzionate v. Kuttner, Réflexions (supra, n. 29), p. 771ss.

${ }^{37}$ Genzmer, op. cit. (supra, n. 30), p. 424.

${ }^{38}$ Kuttner, Réflexions (supra, n. 29), p. 767-769; l'autore precisa in ogni caso (ivi, p. 791) che ciò non vuol dire che tutti i brocarda assurgano a regole generali del diritto: spesso, infatti, la vis dei contraria impedisce una generalizzazione della massima. Quando tuttavia il generale contiene una massima che può essere considerata una regola generale del diritto, i contraria non sono ridotti ad altro che a specialia. Questa interpretazione non convince M. Schwaibold, 'Dolum per subsequentia purgari'. Eine englische Sammlung von Argumenten des romischen Rechts aus dem 12. Jahrhundert, Frankfurt am Main 1985, p. 101.

${ }^{39}$ Weimar, Argumenta (supra, n. 9), p. 98. In questo senso anche Legendre, op. cit. (supra, n. 6), p. 80-81. Schwaibold, Dolum (supra, n. 38), p. 102, propone un ridimensionamento della teoria di Weimar rispetto al ruolo dell'oppositio contrariorum come elemento fondamentale del brocardo, sulla scorta del fatto che nella collezione di Brocardica da lui edita, la cd. Dolum, circa metà degli argomenti non presentano questa opposizione.

${ }^{40}$ Kuttner, Réflexions (supra, n. 29), p. 769-770. Schwaibold, Dolum (supra, n. 38), p. 105, ritiene che la ragione dell'aggiunta delle solutiones sia da ricercarsi un due fattori: da un lato la necessità - in chiave sistematica - di risolvere le antinomie, dall'altro quella eminentemente pratica di utilizzare i principi giuridici del diritto giustinianeo nell'attività forense.
} 
Diversamente Caprioli, prendendo proprio le mosse dalla caratteristica saliente dei contraria, ha visto lo scopo di tale opposizione proprio nella risoluzione delle antinomie e nella necessità di fornire criteri generali di giudizio, che si concretizzerebbe nel ruolo fondamentale della solutio. Ed è in quest'ultima dunque, e non negli argumenta, che per Caprioli bisogna scorgere l'emersione di principi giuridici generali (generalia) ${ }^{41}$. Egli infatti eloquentemente definisce il brocardum come essenzialmente 'ricerca della solutio di una antinomia'42.

Veniamo ora proprio alle suddette enunciazioni contenute nei brocardi, ossia alle massime che essi contengono nella loro impostazione dualistica. Savigny per primo definì i brocarda e le massime in essi esposte 'regole generali del diritto' (allgemeine Rechtsregeln, in accordo con il termine di generalia che era sovente in uso per riferirsi ad essi ${ }^{43}$ ), ossia delle regulae iuris ${ }^{44}$, esaltandone la propensione al trattamento sistematico ${ }^{45}$. La valenza profondamente sistematica dei loro enunciati è stata evidenziata anche da Kuttner, che ne individua le ragioni della nascita nell'esigenza di collegare le norme giuridiche particolari all'enunciazione di principi generali, rispondendo quindi al bisogno di una scienza sistematica del diritto ${ }^{46}$. A questa istanza di sistematicità risponde anche lo sviluppo delle solutiones: per loro tramite i glossatori mettono il mezzo delle massime generali al servizio della conoscenza sistematica del diritto ${ }^{47}$. Weimar invece, dal canto suo, ha messo in discussione la definizione di Savigny delle massime brocardiche come regole generali del diritto: esse sarebbero - sulla scorta delle definizioni coeve - semplici insiemi di argumenta, distinte dalle regulae iuris e caratterizzate proprio dalla loro opposizione dialettica. Mentre insomma i brocardi sarebbero coppie di argomenti opposti senza valenza generale, soltanto con la redazione

\footnotetext{
${ }^{41}$ Cfr. Weimar, Argumenta (supra, n. 9), p. 94 e S. Caprioli, Tre capitoli intorno alla nozione di regula iuris nel pensiero dei glossatori, Annali di storia del diritto, 5/6 (1961/62), p. 221-374, p. 221-226, e in particolare p. 223 n. 2 e p. 225.

${ }^{42}$ Caprioli, op. cit. (supra, n. 41), p. 221.

${ }^{43}$ Riguardo all'uso del termine generalia per riferirsi ai Brocarda, v. ivi, p. 223 n. 5 e Kuttner, Réflexions (supra, n. 29), p. 768 n. 3.

${ }^{44}$ Sul concetto di regula iuris v. Caprioli, op. cit. (supra, n. 41), passim; P. Stein, The formation ot the gloss de regulis iuris and the glossator's concept of regula, in: Atti del Convegno internazionale di studi accursiani (Bologna, 21-26 ottobre 1963), 2, Milano 1968, p. 697-722; Id., Regulae iuris. From juristic rules to legal maxims, Edinburgh 1966; H. F. Jolowicz, Roman regulae and English maxims, in: L'Europa e il diritto romano. Studi in memoria di Paolo Koschaker, 1, Milano 1954, p. 213-223, p. 221s.

${ }^{45}$ Savigny, op. cit. (supra, n. 26), 3, p. 567, 570.

${ }^{46}$ Kuttner, Réflexions (supra, n. 29), p. 767.

${ }^{47}$ Ivi, p. 770.
} 
delle solutiones si assisterebbe allo sviluppo di veri e propri principi giuridici ${ }^{48}$. Lo scopo degli argumenta contrapposti era quindi quello di fornire alla pratica forense e all'insegnamento didattico i materiali necessari per le argomentazioni giuridiche ${ }^{49}$. Il carattere sistematico dei Brocarda (e nello specifico della costruzione di una solutio) è stato evidenziato anche da Caprioli ${ }^{50}$.

In ogni caso, alla luce dei brevi cenni sul genere brocardico, e in particolare - come evidenziato da Caprioli e Weimar - della centralità della solutio rispetto all'affermazione di principi di ordine generale, ciò che in questa sede preme rilevare è che proprio nella formulazione della solutio di Azzone al nostro brocardo in tema di moneta si potrà cogliere la portata realmente innovativa del contributo del giurista bolognese, ossia l'affermazione di una regola giuridica di generale applicabilità.

\section{I Brocarda di Ottone-Azzone in particolare}

Nello specifico, il brocardo di nostro interesse si trova nella collezione presumibilmente compilata da Ottone da Pavia (XII-XIII sec.) ${ }^{51}$ e ripresa da Azzone, il quale aggiunse agli argumenta

\footnotetext{
${ }^{48}$ Weimar, Die legistische (supra, n. 6), p. 237ss; Id., Argumenta (supra, n. 9), p. 91. In questo dibattito, di 'brocardi all'intersezione tra principî e regole (del diritto)' parla Dalbrenta, op. cit. (supra, n. 29), p. 69-71.

${ }^{49}$ Weimar, Argumenta (supra, n. 9), p. 91. Sul ruolo e sulla logica dei brocarda v. anche Dalbrenta, op. cit. (supra, n. 29), p. 79 e ss.

${ }^{50}$ Caprioli, op. cit. (supra, n. 41), p. 225.

51 Per approfondire v. L. Loschiavo, Ottone da Pavia, in DBGI (supra, n. 4), 2, Bologna 2013, p. 1475. Il solo esplicito riferimento ad Ottone è contenuto proprio nel titolo dell'edizione riveduta da Azzone ('Incipiunt generalia que uulgo brocarda dicuntur a domino Otone composita et eorundem discordantium concordia'): v. S. Kuttner, Analecta iuridica Vaticana, in: Collectanea Vaticana in honorem Anselmi M. Card. Albareda, 1 [Studi e Testi, 219], Città del Vaticano 1962, p. 447 e ivi n. 3. Il primo studioso ad aver riconosciuto ad Ottone la redazione di un'opera di brocardi fu M. Sarti, De claris archigymnasii bononiensis professoribus a saeculo XI usque ad saeculum XIV, 1, Bologna 1769, p. 307: 'Ita in Jure Civili Otto Papiensisi Brocharda collegerat, quae recensuit, et supplevit Azo, ut suo loco diximus', citato anche da Savigny, op. cit. (supra, n. 26), 4, p. 384 n. a). Lo stesso Savigny (ivi, p. 384) aveva poi negato che Ottone potesse essere l'autore dell'opera poi riveduta da Azzone, sulla base dell'assenza di fonti in Sarti. Seckel tuttavia propende certamente per la paternità originale estranea ad Azzone, sulla base delle frequenti critiche del giurista bolognese ai Brocarda stessi (Seckel, Distinctiones (supra, n. 8), p. 384 n. 2), e di questa opinione sono anche Weimar (Weimar, Argumenta (supra, n. 9), p. 121) e Kuttner (Kuttner, Réflexions (supra, n. 29), p. 770; Id., Analecta (supra, n. 51), p. 447). Per un resoconto storiografico dell'attribuzione ad Ottone di un'opera di brocardi v. Schwaibold, Wer sucht (supra, n. 6), p. 202-205.
} 
le proprie soluzioni ${ }^{52}$; il tutto fu poi ancora rielaborato dal suo allievo Cacciavillano, che apportò delle ulteriori aggiunte al testo ${ }^{53}$. Nella formulazione originaria ottoniana, i Brocarda rappresentano una delle più antiche opere di questo genere ${ }^{54}$.

I Brocarda di Ottone-Azzone sono dunque un'opera profondamente stratificata e graduale, arricchitasi nel corso del tempo per opera di mani diverse. La recensione più antica dell'opera è stata individuata da Weimar nei cd. Brocardica 'Dolum per subsequentia purgari', sopravvissuti in sei manoscritti ${ }^{55}$. In essi, contrariamente alla recensione di Azzone, le allegazioni sono quasi sempre citate per esteso e il numero dei brocardi e delle rubriche è di molto inferiore rispetto alla recensione finale. A differenza di quanto ritenuto dal Weimar, Mathias Schwaibold ha suggerito che la raccolta Dolum non sia una variante della tradizione ottoniana, ma un'opera di origine inglese che costituirebbe solo il modello preso a riferimento da Ottone per la redazione della sua opera ${ }^{56}$, mentre egli individua la recensione ottoniana nel ms. Vaticano, BAV, Chigi Chigi E.VII.218, fol. 1ra-22vc $(\mathrm{CH} 2) \mathrm{e}$, in una versione leggermente più breve, nel ms. Paris, Bibliothèque Nationale, lat. 4601, fol. 1ra-23rc (P1); quest'ultima raccolta coincide essenzialmente con quella standardizzata poi da Azzone, ma è priva di solutiones ${ }^{57}$.

Avremmo dunque quattro momenti della redazione della raccolta: il modello embrionale costituito da Dolum sarebbe stato ripreso in Italia da Ottone da Pavia (di cui si segnalano solo i due manoscritti suddetti), che avrebbe adeguato le citazioni del Corpus iuris all'uso bolognese ma non avrebbe formulato le solutiones. Rimaneggiando la raccolta di Ottone, Azzone avrebbe aggiunto le sue solutiones delle antinomie, pervenendo così alla formulazione di generalia, cioè princìpi giuridici astratti che rendono sistematicamente coerenti le norme in apparente conflitto fra loro. Infine

\footnotetext{
${ }^{52}$ Genzmer, op. cit. (supra, n. 30), p. 426 e ivi n. 356. Kuttner, Réflexion, (supra, n. 29), p. 770 n. 12, osservò come l'indeterminatezza dei gradi di riscrittura dell'opera abbia reso quasi impossibile stabilire chiaramente quali solutiones siano state aggiunte da Azzone e quali altrimenti; di questo avviso è anche Caprioli, op. cit. (supra, n. 41), p. 223 n. 2. In senso contrario v. Schwaibold, Wer sucht (supra, n. 6), p. 207, 208-209.

${ }^{53}$ Weimar, Argumenta (supra, n. 9), p. 121; Savigny, op. cit. (supra, n. 26), 5, p. 38-40, 76; v. anche Kuttner, Analecta (supra, n.51), p. 447 ss. Sull'individuazione dei manoscritti contenenti le aggiunte di Cacciavillano v. Schwaibold, Wer sucht (supra, n. 6), p. 206-208.

${ }^{54}$ Genzmer, op. cit. (supra, n. 30), p. 425.

${ }^{55} \mathrm{Il}$ cui ms. più antico pare essere ms. Stockholm, Kungliga Biblioteket, B. 683, foll. 58ra-109vb; v. Weimar, Argumenta (supra, n. 9), p. 120.

${ }^{56}$ Per l'edizione critica di Dolum v. Schwaibold, Dolum (supra, n. 38), passim; egli ritiene che l'opera sia stata composta intorno al 1160 in Inghilterra (ivi, p. 133; Id., Wer sucht (supra, n. 6), p. 204-205, p. 207).

${ }^{57}$ Schwaibold, Wer sucht (supra, n. 6), p. 210-212.
} 
Cacciavillano avrebbe ampliato ulteriormente l'opera di Azzone con delle additiones, sia rispetto alle solutiones che alle allegazioni.

La ricostruzione dell'autorialità e il rapporto con Dolum sono ulteriormente complicati dall'esistenza di una recensione intermedia, quale sembra essere quella contenuta nel ms. Leipzig, Universitätsbibliothek, 992 (LE1): anche in essa le leges sono citate per esteso come in Dolum, ma il numero di rubriche e di brocardi è superiore ${ }^{58}$. Weimar, nel suo tentativo di individuare l'opera ottoniana in Dolum, ha visto una stretta correlazione tra quest'ultimo e il codice di Lipsia ${ }^{59}$, che testimonierebbe dunque un passaggio intermedio proprio tra Dolum e Azzone. Schwaibold invece, dal canto suo, ritiene che il manoscritto lipsense non sia accomunabile su base testuale a Dolum, mentre avrebbe maggiori punti di contatto con le successive redazioni dei Brocarda; egli dunque ritiene che si tratti di un testo precedente a Ottone, a cui il giurista pavese potrebbe essersi ispirato per la propria opera ${ }^{60}$. Va infine notato come anche il ms. Paris, Bibliothèque Nationale, lat. 4603 contenga una recensione dei Brocarda che pare più risalente di quella di Azzone, in quanto priva di solutiones e le cui rispettive rubriche coincidono solo in parte ${ }^{61}$.

Ferme restando le difficoltà di una esatta ricostruzione della genesi dei Brocarda, e specialmente del loro rapporto con Dolum e il codice di Lipsia, rispetto a queste ultime recensioni quella di Azzone contiene un numero maggiore di rubriche e si differenzia per la presenza delle solutiones alle antinomie proposte, aggiunte proprio dal giurista bolognese. Le allegazioni sono più numerose e non più citate per esteso, bensì attraverso il rinvio alla fonte. Di più sull'opera sarà detto nelle pagine successive, dove si cercherà di indagare l'evoluzione del testo e analizzare il brocardo di nostro specifico interesse.

III.

Il testo

\footnotetext{
${ }^{58}$ Seckel, Distinctiones (supra, n. 8), p. 384 n. 1; Weimar, Argumenta (supra, n. 9), p. 120.

${ }^{59}$ Weimar, ibidem.

${ }^{60}$ Schwaibold, Wer sucht (supra, n. 6), p. 213-214.

${ }^{61}$ Sul ms. in questione, citato da Kantorowicz come possibile opera di Ottone, v. E. Riezler, Venire contra factum proprium. Studien im römischen, englischen und deutschen Civilrecht, Leipzig 1912, p. 44 n. 2; Schwaibold, Wer sucht (supra, n. 6), p. 204 e ivi, n. 10.
} 


\section{I manoscritti}

Escludendo i fragmenta e gli excerpta, la recensione di Azzone dei Brocardica è conservata in almeno trentasette manoscritti. Per quanto riguarda il passo di nostro interesse, dal conto va escluso il ms. Vaticano, BAV, Ott. lat. 482, che risulta mutilo di alcuni passi62. Dei restanti manoscritti, ho potuto consultarne, direttamente o tramite microfilm, trenta ${ }^{63}$. Una menzione particolare merita il ms. Leipzig UB 992: come si è detto, esso contiene ai foll. 72ra-95rb (LE1) una versione intermedia dei Brocarda, più vicina alla recensione Dolum che a quella Salicta azzoniana, priva di solutiones. Queste ultime, invece, sono state aggiunte, nella recensione a noi nota, ai foll. 96ra-115va (LE2). Vanno menzionati anche: il ms. Paris, BNF, lat. 4601, che riporta i brocardi privi di soluzioni (seppur nella versione finale dei Brocardica Salicta) ai foll. 1ra-21rc (P1), mentre le solutiones sono aggiunte ai foll. 23ra-35vb (P2); e il ms. Vaticano, BAV, Chigi E.VII.218, che riporta i Brocarda due volte: una prima, senza solutiones, ai foll. 1ra-22vc (CH2), e una seconda, completa, ai foll. 37ra-58vb (CH3). Per tali ragioni, si è ritenuto di sdoppiare le sigle dei tre manoscritti summenzionati.

I manoscritti che contengono i Brocarda di Azzone sono tutti miscellanei che ospitano altre opere diverse per tematiche e generi. Per la maggior parte si tratta di opere civilistiche ${ }^{64}$, non mancano

\footnotetext{
${ }^{62} \mathrm{Il} \mathrm{fol} .12 \mathrm{v}$, infatti, si conclude con il penultimo brocardo precedente quello di nostro interesse (Quod actum sit inter contrahentes inspicitur), di cui è citata la penultima allegazione (ff. quod sine manumissione ad libertatem perveniunt $l$. ultima). Dopo di che, alla prima riga del foglio successivo (13r), il testo riprende bruscamente a metà di un diverso brocardo, all'interno della rubrica de actionibus. Manca evidentemente almeno un foglio, contenente sia il brocardo di nostro interesse, sia la successiva rubrica de pactis e l'inizio di quella de actionibus.

${ }^{63}$ I restanti sono: Lambach, Stiftsbibliothek, chart. 160; Metz, Bibliothèques-Médiathèques de la Ville de Metz, 102; München, Bayerische Staatsbibliothek, clm. 3507; La Seu d'Urgell, Arxiu Capitular d'Urgell, 2022; Toledo, Biblioteca de la Catedral, 39-28; Tours, Bibliothèque municipale, 657. Come segnalato da Ernst, The Glossators' (supra, n. 3), p. 234 n. 59, la sola solutio del brocardo è poi presente anche in una glossa anonima nel ms. Torino F.II.14, fol. 109r.

${ }^{64}$ Per il contenuto dettagliato dei mss. v. G. Dolezalek, Manuscripta Juridica, www.manuscripts.rg.mpg.de, consultato il 6 settembre 2018. Particolarmente interessante è al riguardo WO, che contiene anche estratti del Digestum novum.
} 
tuttavia alcuni contenuti di matrice canonistica ${ }^{65}$ e di diritto feudale ${ }^{66}$. Per quanto riguarda le opere di diritto civile, vi sono numerosi tractatus ${ }^{67}$; grande rilievo ha la rilevante presenza di prodotti appartenenti a un genere altamente sistematico come le distinctiones ${ }^{68}$. Molte sono poi le opere appartenenti a generi letterari che utilizzano un metodo dialettico affine a quello dei brocarda, come le quaestiones $^{69}$ e i notabilia ${ }^{70}$, mentre solo in due casi compare il Libellus disputatorius ${ }^{71}$. Si trovano infine con una certa frequenza opere di forte connotazione pratica e forense come gli ordines iudiciorum $^{72}$.

\section{Le edizioni}

Dopo aver esaminato la tradizione manoscritta, è necessario qualche breve accenno alle edizioni a stampa. È infatti su queste che si è basata gran parte dell'indagine scientifica sui brocardi,

${ }^{65}$ Quaestiones iuris canonici (AV, che contiene anche opere di teologia come una expositio Bibliae, e SI); Apparatus ad Decretalium Librum Sextum 5.13 de regulis iuris di Dino del Mugello (BO); Summa Decretalium X.4 de sponsalibus et matrimoniis di Giovanni d'Andrea (BO); Florilegium iuris canonici (BRU); abbreviatio del Decretum Gratiani (BRU); Libelli in iure canonico di Roffredo Beneventano (MA); una lettera ad Onorio III dal Capitulum Senonense (SI); notabilia al Decretum (SI); Casus Decretalium di Johannes de Deo (VA1); formule della giurisdizione papale delegata in Inghilterra (VA1); Summa Decretalium di Giovanni da Petesella (VA1), i Brocarda di Damaso (CA2), costituzioni papali e canoni conciliari (MU1).

${ }^{66}$ Libri feudorum (CH1).

${ }^{67}$ La quantità e l'eterogeneità non ne rende possibile un'elencazione completa. A titolo esemplificativo basti considerare la ricorrente presenza del Tractatus de exceptionibus di Pillio (BAM2, CH1, O1, VA1), Tractatus de praeparatoriis litium (BRU, O1, P3, SI) e altri numerosi trattati di materia processuale (O1, CH2-3, P1-2, P3, SI, VA1); vi sono poi diversi tractatus di altri giuristi, tra i quali i più ricorrenti sono quelli di Benedetto da Isernia (O1, CH2-3) e Alberico di Porta Ravennata (SI, CH2-3).

${ }^{68}$ Sono presenti, tra le altre, distinctiones di Ugolino (BAM2, BRE, CA1, DU, MA, P1-2, P3, VA2), Ugo di Porta Ravennata (CH1, CH2-3, O1), Alberico di Porta Ravennata (VA2), Pillio (CH1, O1, P1-P2, CH2-3), Azzone (BRU, O1, $\mathrm{CH} 2-3$ ), Giovanni Bassiano (CH1, CH2-3, O1, P1-P2, VA2), Piacentino (CH1) e Ottone da Pavia (CH1).

${ }^{69}$ Quaestiones 'Insolubilia' di Ugolino (BAM2, P3, SI, VA2, CH2-3); Quaestiones sabbatinae di Pillio (BAM2, CA1, P3, SI, VA2); Quaestiones di Azzone (BAM2, MA, P3, SI); Quaestiones di Roffredo (BAM2, CA1, MA, SI); Quaestiones di Giovanni Bassiano (SI);

${ }^{70}$ Notabilia Codicis Justiniani (BAM2, SI), Notabilia Authentici (BAM2), Notabilia iuris civilis (BAM2), Notabilia 'ex omni corpore iuris' (P3, SI), Notabilia Decreti (SI), Notabilia Digestorum (SI), Notabilia Infortiati (W1).

${ }^{71}$ Come il Libellus Disputatorius (BRU, SI), i Brocardica 'Dolum' (WO) e i brocarda canonistici di Damaso (CA2).

72 Ordo iudiciorum 'Invocato Christi nomine' (BAM2), Ordo iudiciarius di Tancredi da Bologna (BAM2, BRE, R, VA1), Ordo iudiciorum di Pillio (BRU, P3), Ordo iudiciarius di Riccardo Anglico (BRU), Ordo iudiciorum di Azzone (CH1), Ordo iudiciarius di Pietro Ispano, Ordo iudiciorum 'Olim' di Ottone (SI, WO), Ordo iudicarius di Bartolomeo da Brescia (DU). 
mentre - come detto - manca tutt'ora un'edizione critica che risalga ai manoscritti. Si è visto sopra come anche lo studio di Taeuber sul brocardo di nostro interesse si basi fondamentalmente sull'edizione del 1567 di Caspar Hervag. L'editio princeps, pubblicata nel 1557, è andata perduta ${ }^{73}$. A questa seguirono altre quattro edizioni: Venezia 1566, dove la raccolta di brocardi, che conta 95 rubriche, è introdotta in appendice alla Summa Codicis e alla Summa Institutionum (rist. 1581, 1593, 1610; rist. anast. Frankfurt am Main 2008) ${ }^{74}$; Basilea 156757, 104 rubriche; Napoli 1568 ${ }^{76}$, 97 rubriche (rist. anast. CGIC, IV.3, Torino 1967); Torino 157777, 106 rubriche. Mentre l'edizione basilense pare essere stata pubblicata nella totale ignoranza di quella veneziana dell'anno precedente, l'edizione di Torino (scoperta da Kuttner) e quella di Napoli sono edizioni miste, basate su quelle di Venezia e Basilea $^{78}$.

Queste edizioni cinquecentesche vanno considerate nel clima culturale che caratterizza il loro tempo. Di conseguenza, i diversi editori sono intervenuti più volte sul testo, specialmente nel tentativo di adeguare le allegazioni basate sulla Vulgata del Corpus iuris al testo giustinianeo così come emendato dagli umanisti. Su tutti un esempio: il testo al $\S 41$ della nostra edizione critica 'Sic enim dixerat: quod supererit ex hereditate tempore mortis, vel, que in ea erit, cum moriar', nell'edizione napoletana del 1568 diviene 'Sic enim dixit Philargium puerum, et Agatheam ancillam, qui mei erunt cum moriar' ${ }^{79}$, nel tentativo di ripristinare la fedeltà al passo ivi citato del Digesto (D. 35.1.28.1). È evidente come questi interventi compromettano la possibilità per lo studioso di basarsi sulle edizioni moderne per conoscere il testo originario, ingenerando talvolta confusione o errori nella corretta comprensione delle allegazioni al Corpus.

\footnotetext{
${ }^{73}$ v. A. Gouron, Introduzione, in: Azzone, Summa codicis (Institutionum et Digestorum) \& Brocardica, [rist. anast. di Id., Aurea Brocardica, Venezia 1581], a cura di A. Gouron, Frankfurt am Main 2008, p. IX-XX; A. Converso, Introduzione, in: Azzone, Azonis Brocarda [CGJC, 4.3], Torino 1967 [rist. anast. di Id., Brocardica aurea, Napoli 1568], p. III.

${ }^{74}$ v. Azzone, Summa codicis (Institutionum et Digestorum) \& Brocardica (supra, n. 73).

${ }^{75}$ v. Id., Brocardica, siue Generalia iuris, Basel 1567.

${ }^{76}$ v. Id., Azonis Brocarda (supra, n. 73).

77 v. Id., Brocardica, seu generalia iuris, Torino 1577.

${ }^{78}$ Weimar, Argumenta (supra, n. 9), p. 121 n. 124. Sulle edizioni a stampa v. anche Kuttner, Analecta (supra, n.51), p. 446 n. 5, Savigny, op. cit. (supra, n. 26), 5, p. 39ss., nonché Converso, op. cit. (supra, n. 73), p. I-III ed E. Landsberg, Die Quaestiones des Azo. Zum ersten Male aus den Handschriften herausgegeben, bevorwortet und mit Noten versehen, Freiburg 1888, p.108-109.

${ }^{79}$ Azzone, Azonis Brocarda (supra, n. 73), p. 73; un intervento simile è presente sull'ed. veneziana: cfr. Id., Summa codicis (Institutionum et Digestorum) \& Brocardica (supra, n. 73), coll. 13-14.
} 


\section{Indagine sull'origine del testo}

Venendo all'analisi più da vicino del nostro brocardo (Eadem mensura vel moneta debetur, que erat tempore contractus), sono necessarie alcune precisazioni rispetto alla sua tradizione. Esso è infatti assente nella più antica recensione dei Brocarda, la cd. Dolum ${ }^{80}$, come anche in quella contenuta nel ms. Paris, Bibliothèque Nationale, lat. 4603; compare tuttavia, senza la solutio $^{81}$, nella redazione intermedia contenuta in LE1, al fol. 76rv. Confrontando le due recensioni del brocardo, si può notare come la recensione lipsiense contenga un numero molto più esiguo di allegazioni rispetto alla recensione finale: soltanto sei, di cui tre pro e tre contra (ossia quelle presenti nella prima parte del $\S 4$ e nei $\S \S 5,7,24,25$ e 27 del nostro testo). Si tratta rispettivamente di D. 2.11.11, D. 2.13.8.1, D. 12.1.3 e D. 35.1.28, D. 28.2.4, D. 12.1.22. È significativo notare come già nella versione intermedia di Lipsia compaiano tra le allegazioni quelle che nella tradizione giuridica medievale furono le sedes materiae in tema monetario: la cd. lex Cum quid (D. 12.1.3) e la lex Vinum (12.1.22), sulle quali si tornerà più avanti.

Nello stadio finale della redazione dei Brocarda, invece, il numero di allegazioni è di molto superiore: tra le varie lezioni se ne contano ben 33, di cui 19 pro e 14 contra, tra cui anche una serie di rimandi interni agli stessi Brocarda. Anche le fonti delle citazioni si sono ampliate: mentre LE1 contiene esclusivamente passi del Digesto, la recensione di Azzone contiene numerosi rimandi al Codex, uno all'Authenticum e due anche al Decretum Gratiani.

\section{Divergenze nelle allegazioni}

Sempre riguardo alle allegazioni, bisogna considerare come queste rappresentino una parte essenziale del testo giuridico e del genere brocardico. Attraverso di esse, il giurista non solo raccoglie in modo sistematico i testi normativi a favore e contro un determinato argomento di diritto, fornendo così ad esso il fondamento della sua legittimazione, ma - come ha osservato Menzinger ${ }^{82}$ - fa costante riferimento alle categorie giuridiche romane.

È dunque proprio sulle allegazioni che è necessario intraprendere particolari indagini per quanto riguarda il nostro brocardo, e sono proprio queste a costituire la parte più problematica della ricostruzione testuale. Il confronto tra i diversi manoscritti evidenza un altissimo numero di

\footnotetext{
${ }^{80}$ Per un elenco delle rubriche, v. Schwaibold, Dolum (supra, n. 38), p. 163-167.

${ }^{81}$ Questa è contenuta al fol. 100r del codice di Lipsia (LE2).

${ }^{82} \mathrm{~S}$. Menzinger, Riflessioni sul rapporto tra autore e testo nella produzione giuridica medievale, Historia et ius, 11 (2017), p. 1-18, p. 8.
} 
deviazioni nelle allegazioni tra una lezione e l'altra. Bisogna innanzitutto distinguere tra le varianti che si verificano solo una volta - le quali per lo più rappresentano fenomeni isolati che non aiutano a chiarire la storia del testo - e quelle varianti che invece appaiono come ricorrenti e che sembrano significative. I fenomeni più frequenti sono l'assenza di determinate allegazioni in più manoscritti, la ricorrenza di determinati errori o l'inversione di certe parole all'interno di una frase. Altro fenomeno di una certa rilevanza è un diverso ordine delle allegazioni ricorrente in più lezioni.

In ogni caso, si tratta di episodi che presi singolarmente possono dirci poco e non consentono di trarre conclusioni di ampia portata sulla storia del brocardo; tuttavia, esaminando in maniera incrociata determinate varianti nelle allegazioni, è possibile distinguere due gruppi più o meno coerenti di manoscritti. Il primo, che chiameremo G1, comprende i manoscritti BRU, CH1, CH2, CH3, MI, MZ, O1, O2, P1, W1, WO; il secondo, G2, racchiude i mss. AV, BAM1, BAM2, BO, BRE, CA1, CA2, DU, LA, MA, MU1, P3, R, SI, TO, TR, VA1, VA2 ${ }^{83}$. Il ms. W2, invece, si presenta molto più scarno nelle allegazioni rispetto al testo dei due gruppi, di conseguenza ci sembra che non possa essere classificato in uno di questi; ciò nondimeno, presenta in più punti una qualche affinità con il ms. O1. Da questa sommaria differenziazione sono stati ovviamente omessi i mss. LE2 e P2, in cui è presente solo la solutio. In quest'ultima parte, infatti, le varianti tra le diverse lezioni sono decisamente meno significative e per lo più sembrano derivare da comuni errori di trascrizione.

Scendendo maggiormente nel dettaglio, possono essere fatte le seguenti osservazioni: il gruppo che abbiamo denominato G1 presenta al suo interno un notevole grado di eterogeneità e un più alto numero di varianti rispetto a G2. In particolare, i mss. O2 e W1 presentano numerose affinità testuali tra di loro, e in diversi punti divergono dal resto del gruppo o da entrambi i gruppi, come nei $\S \S 10,15$ e 29; si tratta anche degli unici a recare il $\S 28.1$. All'interno di G1, va fatta una menzione particolare del ms. $\mathrm{CH} 1$, in cui il testo si presenta corretto da una mano che è intervenuta successivamente integrando lacune e correggendo errori (es. aggiunta di '1. Qui autem $\S$ illud' al $\S 4$ e di ' $§$ I' al $\S 5)$.

\footnotetext{
${ }^{83}$ La suddivisione tra G1 e G2 corrisponde grosso modo (e con l'eccezione di O2) a quella tracciata da Schwaibold, Wer sucht (supra, n. 6), p. 206 nn. 25-26, tra i manoscritti in cui compare rispettivamente la versione dei Brocarda di Azzone e quella con le aggiunte di Cacciavillano. Il nostro lavoro non lascia spazio a un'indagine più approfondita sull'individuazione delle aggiunte da parte di quest'ultimo, tuttavia - attenendoci alla suddivisione di Schwaibold e alla sua considerazione che l'allievo avrebbe aggiunto anche numerose allegazioni (ivi, p. 208) - con un certo grado di cautela potremmo spiegare la differenza significativa tra le due versioni - e specialmente il maggior numero di allegazioni in G2 - come riferibile alla mano del Cacciavillano.
} 
Nei manoscritti del gruppo G1 - oltre che in W2 - ricorrono inoltre anche delle variazioni nell'ordine in cui le allegazioni sono riportate. Secondo quest'ultimo criterio, i manoscritti in G1 possono essere suddivisi come segue: un primo gruppo che comprende $\mathrm{CH} 2, \mathrm{O} 2, \mathrm{P} 1, \mathrm{~W} 1$; un secondo composto da CH1 e MI (che condivide anche con BRU e WO l'ordine dei $\S \S 3,16,4$, mentre questi ultimi due a loro volta condividono l'ordine dei $\S \S 13,15$ e 16); un terzo gruppo, che include tutti gli altri, i quali presentano forti irregolarità e non hanno alcuna comunanza. Come già detto, in G2 invece sono maggiormente rari i casi in cui i singoli testi si discostano dal modello comune, come meno frequenti sono le varianti. Una posizione particolare è occupata da P3, unico manoscritto a riportare i $\S \S 24.1$ e 24.2 .

A voler riassumere le principali differenze tra i due gruppi di manoscritti, si può osservare come quelli affini a G1 contengano un minor numero di allegazioni: mancano del tutto i $\S \S 2,6,12$, 13, 17-21, 23; risultano inoltre monche le allegazioni ai $\S \S 4,5,7,24,28$. Per contro, in G2 sono assenti i $\S \S 10$ e 15 . Le varianti testuali più significative tra i due gruppi sono riscontrabili nei seguenti paragrafi: $\S 1$, in cui G1 colloca il verbo alla fine della frase; 8, in cui G2 reca 'bonitatis', mentre G1 riporta, con leggere varianti, la lezione 'ne inst.'; § 11, dove G2 recita 'filium' e G1 'filii', e poi ancora rispettivamente 'testator' e 'testamento non aliter'; $\S 16$, ove G2 riporta senza eccezioni 'ma.', mentre G1 dà (salvo tre varianti erronee) la lezione corretta 'naviculariorum'; $§ 22$, dove G1 recita 'minorve' invece di 'sive minor'; $§ 25$, dove in luogo di 'placet' G1 reca 'hii placet'.

Come già detto, la sistematicità e l'occorrenza delle varianti si riduce drasticamente nella solutio, in cui queste coinvolgono un numero minore di manoscritti e sembrano rispondere più a errori materiali dei copisti che a differenze significative nella tradizione testuale. Una certa omogeneità nelle varianti rispetto alle famiglie è forse riscontrabile solamente al $\S 35$, nell'ordine del binomio mensura/moneta, e al $\S 38$, dove G2 riporta 'in bonis debitoris', mentre in G1 manca la prima parola.

\section{Identificazione dei filoni e ricostruzione testuale}

Malgrado quanto esposto sulla ricorrenza di alcune varianti e l'individuazione di due gruppi nella tradizione manoscritta del nostro brocardo, sono necessarie alcune precisazioni. Indubbiamente uno degli scopi di un'edizione critica e della collazione di un testo dovrebbe essere quello di identificare i filoni della tradizione ${ }^{84}$. Ciò nonostante, ritengo che questo principio di critica del testo non possa essere facilmente applicato a un'opera giuridica particolare come quella di Azzone-Ottone,

\footnotetext{
${ }^{84}$ v. H. Kantorowicz, Introduzione alla critica del testo. Esposizione sistematica dei principi della critica del testo per filologi e giuristi, Roma 2007, in particolare p. 26ss.
} 
e per diversi motivi. Innanzitutto, i Brocarda sono un'opera estremamente stratificata nel corso del tempo, e strutturata su più livelli: le singole rubriche, la serie di allegazioni e le solutiones. Ognuno di questi aspetti dovrebbe essere esaminato approfonditamente e rende difficoltosa la costruzione di uno stemma codicum secondo i criteri comuni. Per lo stesso motivo, inoltre, qualunque tentativo di ricostruzione delle famiglie dovrebbe coinvolgere l'analisi dell'intero lavoro di Azzone e non solo il breve brocardo oggetto del presente studio.

Sembrerebbe poi imprescindibile, ai fini della ricostruzione dei filoni testuali, tenere conto delle particolari modalità di trasmissione e riproduzione del testo azzoniano. Numerosi errori testuali nel nostro brocardo sembrano derivanti dalla evidente incomprensione delle abbreviazioni da parte del copista, e dunque da tentativi di scioglimento errati. Una considerazione, questa, che dà l'idea di un testo sovente riprodotto e diffusosi mediante copisti professionisti e non tramite giuristi coscienti del contenuto dell'opera. È certo, infatti, che si adottò il sistema della riproduzione per exemplar e pecia, come descritto nel saliente lavoro di Soetermeer ${ }^{85}$; ciò è confermato anche dalla presenza dei Brocarda di Azzone tra i testi inclusi negli elenchi di tassazione degli stazionari ${ }^{86}$. Pertanto, mi asterrò da ulteriori tentativi in tal senso, non essendo uno studio di questo tipo nella portata della presente indagine.

Sulla base delle medesime considerazioni, ritengo che nella redazione della presente edizione si debba rinunciare all'idea dell'esistenza di un unico testo autoriale originario. E non solo per la sovrabbondanza di varianti, per il sistema di riproduzione in pecie e per la formazione graduale dell'opera azzoniana - che pure di per sé paiono motivi rilevanti -, ma anche alla luce della necessità di un ripensamento radicale del rapporto tra testo e autorialità; ripensamento che proprio le caratteristiche sopra evidenziate rendono opportuno. Come evidenziato da Menzinger, infatti, il tradizionale taglio biografico, che da Savigny in poi ha caratterizzato la storia della letteratura giuridica medievale, ha talvolta indotto a trascurare l'evidente estraneità dell'idea moderna di "autore" alla mentalità scolastica medievale, e di conseguenza il peculiare atteggiamento dei giuristi medievali di fronte alle opere che divulgavano sotto il proprio nome dopo averle riprese da altri ${ }^{87}$. Tale particolare rapporto tra autore e opera è particolarmente evidente proprio nei Brocarda azzoniani, considerando la loro origine come revisione, da parte del giurista bolognese, di un testo precedente di diverso autore. Che i brocarda si rivelino poi un genere letterario intrinsecamente collettivo e aperto, è rivelato inoltre non solo dai continui rifacimenti, dalle recensioni multiple e

\footnotetext{
${ }^{85}$ F. Soetermeer, Utrumque ius in peciis. Aspetti della produzione libraria a Bologna fra Due e Trecento, Milano 1997.

${ }^{86}$ Ivi, p. 350; v. anche Savigny, op. cit. (supra, n. 26), 5, p. 39.

${ }^{87}$ Menzinger, op. cit. (supra, n. 82), p. 1-10.
} 
dalla formazione alluvionale delle opere, ma dalla stessa attitudine fortemente dialettica del genere, in stretta connessione con la rilevanza e le finalità pratiche che esso assumeva. Non intendo con ciò mettere in discussione in toto la possibilità e tanto meno l'opportunità di una ricostruzione filologica del testo, ma rimarcare la necessità di tenere conto di quanto detto nella compilazione dell'edizione critica.

\section{Note sulla presentazione dell'edizione}

Da quanto finora detto discendono alcune considerazioni sui criteri per la selezione del testo. Innanzitutto, dato l'alto numero di manoscritti, si è scelto di procedere con un apparato negativo del testo $^{88}$. In secondo luogo, va tenuto conto dell'impossibilità dell'individuazione di criteri all'interno della tradizione in base ai quali scegliere un manoscritto principale e considerare gli altri come varianti. Di conseguenza, nel caso di impossibilità di risalire alla variante corretta di un determinato passo, ho seguito la lezione più comune: è il caso ad esempio del $\S 4$, ove in luogo della parola corretta quempiam, non utilizzata da nessun manoscritto, mi sono attenuto alla variante più diffusa quam.

Inoltre, come già detto in precedenza, alcuni manoscritti sono stati qui indicati con una doppia sigla in virtù delle loro specificità: così il ms. Vaticano, BAV, Chigi.E.VII 218, che ai foll. 5v e 42r contiene due diverse lezioni dei Brocarda, è citato rispettivamente come $\mathrm{CH} 2$ e $\mathrm{CH} 3$; il ms. Leipzig, Universitätsbibliothek, 992, che al fol. 76rv riporta una recensione più risalente mentre al fol. 100r reca la solutio di Azzone, è indicato rispettivamente come LE1 e LE2 ${ }^{89}$; allo stesso modo il ms. Paris, che reca al fol. 5r le allegazioni e al fol. 26r la solutio, ha le sigle P1 e P2.

Infine, come si è già detto e come si vedrà meglio più avanti nel caso di specie, le allegazioni costituiscono un aspetto fondamentale della struttura del brocardo, e non semplici rimandi a un testo esterno. Di conseguenza, nella scelta del luogo dell'edizione critica in cui inserire le citazioni si è scelto di distaccarsi dallo stile tradizionale proposto da Kuttner ${ }^{90}$ e seguire il suggerimento di

\footnotetext{
${ }^{88}$ Vale a dire riportando nel testo la lezione seguita, senza ripetere in nota le fonti che la testimoniano, ma soltanto le varianti: v. S. Kuttner, Notes on the presentation of text and apparatus in editing works of the decretists and decretalists, Traditio, 15 (1959), p. 452-464, p. 458ss.

${ }^{89}$ Per questa ragione, e per la profonda divergenza tra il testo contenuto in LE1 e la nostra recensione dei Brocarda, si è deciso di non procedere alla collazione di LE1 nell'edizione critica, ma di includervi soltanto la solutio contenuta in LE2. ${ }^{90}$ Kuttner, Notes (supra, n. 88), p. 461-464.
} 
Menzinger ${ }^{91}$, inserendole direttamente all'interno del testo tra parentesi, al fine di rimarcare al lettore l'essenzialità delle allegazioni nel testo del giurista medievale.

IV.

Il contenuto

\section{Gli argomenti}

Il nostro brocardo, secondo lo schema tipico del suo genere, si apre con due affermazioni contrapposte, ognuna delle quali corredata da una serie di allegazioni a sostegno, contenute nel Corpus iuris civilis, nel Decretum di Graziano o all'interno degli stessi Brocarda. Le due affermazioni riguardano il tema centrale dell'elaborazione giuridica medievale rispetto alla moneta, ossia la misura da utilizzare per la restituzione di monete in caso di obbligazioni pecuniarie protrattesi nel tempo, qualora sia intervenuta una variazione del valore monetario nel periodo che intercorre fra il contratto obbligatorio e l'adempimento. I due argomenti contrapposti sono rispettivamente: 'eadem mensura vel moneta debetur, que erat tempore contractus' e 'superveniens moneta vel mensura debetur, sive maior sive minor sit'.

Il principio sostenuto dal primo argomento è dunque quello del tempus contractus, in base al quale il debitore è tenuto a fare riferimento al momento della stipulazione contrattuale come criterio temporale per determinare il quantum della controprestazione. Precedenti in questo senso vi erano già stati nelle menzionate quaestiones di Bulgaro e Pillio ${ }^{92}$, nelle quali tuttavia per individuare il momento di perfezionamento del negozio si fa meno genericamente riferimento al tempus dationis, considerando dunque solo il caso di contratti reali che prevedono la datio, come il mutuum, e non anche i contratti consensuali, per il cui perfezionamento basta la volontà delle parti. La ragione del rilievo del tempus contractus è proprio la sua generale coincidenza con il momento di formazione del consenso, e dunque la rilevanza da un punto di vista equitativo e rispettoso della volontà delle parti.

Si tratta di un criterio che, nel caso maggiormente frequente di sopravvenuto indebolimento della moneta, tutela l'affidamento del creditore: questi, infatti, in caso ad esempio di dimezzamento

\footnotetext{
${ }^{91}$ Menzinger, op. cit. (supra, n. 82), p. 7-8.

92 v. supra, nn. 23 e 25
} 
della quantità d'argento contenuta in un denaro, avrà diritto alla restituzione di tot denari secondo la vecchia moneta (di maggiore valore), e quindi alla medesima quantità d'argento da lui prestata, indipendentemente dal fatto che la quantità d'argento contenuta in un denaro corrente sia inferiore. Siamo dinanzi quindi a un'interpretazione sostanzialistica e non nominalistica dell'unità monetaria/di misura stabilita nel contratto, che tenga conto della volontà originaria delle parti.

Il secondo e opposto argomento, invece, sostiene il principio del tempus solutionis inspicitur, in base al quale il debitore è tenuto a determinare la moneta da restituire esclusivamente in base alle condizioni esistenti al momento dell'adempimento. È evidente come in caso di sopravvenuto indebolimento della moneta questa interpretazione cagioni un danno al creditore e un corrispondente vantaggio per il debitore, il quale potrà liberarsi e adempiere pagando una quantità inferiore di metallo prezioso rispetto a quella ricevuta o pattuita, a fronte della medesima quantità nominale di monete.

\section{Le allegazioni}

Veniamo ora più da vicino alle singole allegazioni contenute nel brocardo. Esse sono per lo più inerenti questioni giuridiche sorte da cambiamenti intervenuti nel tempo, o relative a obbligazioni di durata. Vediamo alcuni esempi.

Tra le leges citate a favore del principio del tempus contractus vi è Nov. 115, che stabilisce come le decisioni giudiziarie in appello vadano prese in conformità alle leggi in vigore al tempo in cui è stata emessa la sentenza impugnata e non secondo quelle promulgate successivamente ${ }^{93}$. $\mathrm{O}$ ancora D. 2.11.12, che nel caso di aestimatio giudiziaria stabilisce che questa debba essere commisurata all'interesse dell'attore al tempo in cui avrebbe dovuto avvenire la comparizione, e non a quello in cui si agisce. Sempre rispetto alla determinazione degli interessi, D. 2.13.8.1 afferma che questa vada effettuata secondo la loro entità al momento in cui il pretore ha intimato l'esibizione dei conti, e non nella misura dell'interesse attuale. D. 21.2.13, invece, stabilisce che in caso di evizione la valutazione qualitativa del bene debba riferirsi al momento della vendita e non a quello dell'evizione stessa. Vi sono poi allegazioni di norme testamentarie, come D. 32.1.34.1, in base a cui un erede a cui è stata data facoltà di prendere tra i beni del testatore dei crediti a sua scelta, possa scegliere solo tra quelli presenti nel registro dei crediti al tempo della redazione del testamento; oppure ancora D. 32.1.41.4, per cui se il de cuius intenda lasciare a un legatario cinque servi di meno

\footnotetext{
${ }^{93}$ Il riferimento a Nov. 115 è presente anche nel più antico testo a noi noto che affronta il problema della variazione delle unità di misura nel corso del tempo, ossia la quaestio dello staio Bolognese affrontata da Bulgaro: v. supra, n. 23.
} 
di sette anni, l'età di questi vada valutata in base al tempo del legato e non a quello della morte ${ }^{94}$. Di particolare rilievo sembra essere infine C. 1.14.7, poiché non attiene al tempo di determinazione di una somma pecuniaria, bensì all'efficacia della legge nel tempo. Il passo stabilisce infatti che le leggi e le costituzioni possono regolare esclusivamente negozi futuri, senza alcuna vincolatività retroattiva, a meno che ciò non sia stabilito espressamente e per negozi pendenti; sull'importanza di tale principio torneremo più avanti.

Per quanto riguarda invece le allegazioni al principio del tempus solutionis, anche queste fanno riferimento alla rilevanza giuridica della successione di situazioni diverse nel tempo, chiaramente dal punto di vista opposto. Anche in questo caso diverse leges riguardano la successione mortis causa. Così ad esempio D. 35.1.28 stabilisce che, nel caso in cui il de cuius lasci alla moglie una schiava di nome Agathea, ma poi la venda prima di morire e compri un'altra schiava con lo stesso nome, la moglie erediterà quest'ultima. D. 28.2.4, invece, stabilisce che nel caso di istituzione di un erede postumo, possa considerarsi tale anche l'erede nato da una moglie diversa rispetto a quella del tempo dell'istituzione. E ancora D. 20.1.34, nel caso in cui un debitore dia in pegno le merci contenute in una bottega e poi muoia, stabilisce che il creditore possa soddisfarsi su tutte le merci ivi trovate al momento della morte del debitore e non solo su quelle presenti al momento del pegno. Anche qui abbiamo delle norme relative ai tassi di interesse: C. 4.32.27 stabilisce che coloro che abbiano stipulato un tasso di interesse più alto di quello successivamente fissato per legge, vedano ridotto il proprio tasso in accordo con quanto previsto al tempo dalla legge, a partire dal momento della sua promulgazione.

\section{Le sedes materiae}

Tra le allegazioni, un'attenzione particolare meritano due diversi passi del Digesto, poiché costituiranno le sedes materiae della questione monetaria per tutta la scienza giuridica medievale. Entrambi - come si vedrà - al tempo del nostro brocardo appartengono già al bagaglio giuridico utilizzato dai glossatori in tema monetario, ed entrambi compaiono già tra le poche leges citate dalla recensione intermedia LE1.

\subsection{La lex Cum quid}

\footnotetext{
${ }^{94}$ Le ultime due leges citate, D. 32.1.34.1e D. 32.1.41.4, compaiono anche tra le allegazioni a favore del creditore nella già citata quaestio di Pillio Quidam creditor Lucenses.
} 
Il primo è la cosiddetta lex Cum quid (D. 12.1.3), un passo di Pomponio riguardante i debiti in natura derivanti da mutuum. Esso prevedeva il divieto per il debitore di restituire cose di altro genere o di minore qualità, come ad esempio vino nuovo al posto di quello vecchio: riguardava dunque originariamente le merci, come si evince dall'esempio del vino ${ }^{95}$.

Di primo acchito potrebbe stupire che i glossatori, invece di fare riferimento ai testi del Corpus iuris civilis in tema di moneta, citino un passo che ha a che fare con i beni in natura. Certo, la lex Cum quid si riferiva a un caso di mutuum, fattispecie applicabile alle obbligazioni pecuniarie, ma nel diritto romano il passo di Pomponio riguardava tutti i tipi di mutuum tranne quelli monetari. Ciò potrebbe far sembrare incomprensibile la scelta dei glossatori di estendere la lex Cum quid alle obbligazioni pecuniarie. Tuttavia, conducendo una più approfondita analisi, la scelta è perfettamente coerente con la concezione giuridica di moneta propria del tempo. L'impostazione romana classica infatti, tipica di un'economia perfettamente monetizzata e di un sistema monetario stabile, era difficilmente compatibile con il contesto economico del XII secolo, caratterizzato da una moneta instabile e frequentemente alterata, la quale non circolava su base fiduciaria ma era invece costantemente pesata e accettata in ragione del suo intrinseco ${ }^{96}$. I giuristi dell'età di mezzo alle prese con la concezione della moneta si trovarono quindi ad affrontare il compito di dover adeguare il dettato dei testi giustinianei alla diversa realtà del loro tempo, al fine di plasmarne un diritto vivo e rispondente alle esigenze della società coeva. Essi, dunque, considerarono la moneta non in base al suo valore nominale, ma al suo valore intrinseco, ossia alla quantità di metallo prezioso contenuta. In questa ottica la moneta non era altro che una merce, un pezzo d'argento coniato e valutato in base al suo contenuto metallico ${ }^{97}$.

\footnotetext{
${ }^{95}$ Sull'argomento v. anche Taeuber, op. cit. (supra, n. 3), p. 124. Il testo migliore per la comprensione della lex Cum quid come regola generale per il mutuo è forse contenuto in un passo di Odofredo De Denari, In undecim primos Pandectarum libros < in secundum Digesti veteris>, Lyon 1552 (rist. anast. in Id., Lecutra super Digesto Veteri, 2 [Opera iuridica rariora, 2.2], Bologna 1968), fol. 5r, riportato da Taeuber, op. cit. (supra, n. 3), p. 124 n. 347.

Il principio contenuto in D. 12.1.3 è pervenuto fino ai giorni nostri, conservato nei moderni codici civili di derivazione romanistica: così nel codice civile francese relativamente al prestito di beni consumabili (Code civil, art. 1892), in quello italiano riguardo al mutuo (Codice civile, art. 1813) e nel contratto di prestito del codice tedesco, con espressa esclusione della moneta (Bürgerliches Gesetzbuch, art. 607).

Sulla lex Cum quid v. anche Sargent, Velde, op. cit. (supra, n. 1), p. 75-76. Taeuber, op. cit. (supra, n. 3), p. 125-127, si preoccupa di accertare che il tenore del testo della lex Cum quid noto ai glossatori - ossia quello della cosiddetta Vulgata - non contenesse deviazioni o errori che avrebbero potuto ingannare i giuristi nell'interpretazione del passaggio.

${ }^{96}$ T. Ascarelli, La moneta. Considerazioni di diritto privato, Padova 1928, p. 3, 5; Bloch, op. cit. (supra, n. 1), p. 38.

${ }^{97}$ Sulla concezione della moneta-merce, v. Ernst, The Glossators' (supra, n. 3), p. 226-227; Sargent, Velde, op. cit. (supra, n. 1), p. 4;
} 
Se dunque per i glossatori la moneta era una merce, ossia un bene fungibile che viene pesato e misurato, non deve stupire che ad essa applicassero la disciplina del mutuum, ossia l'istituto giuridico previsto per i beni fungibili ${ }^{98}$. Estendendo alla moneta la disciplina giuridica delle merci, è conseguenza naturale che il mutuo di denaro fosse equiparato al mutuo di $\operatorname{cose}^{99}$. È in ossequio a questa impostazione che essi applicano alle obbligazioni pecuniarie una norma che parla chiaramente di beni in natura. L'esempio del vino previsto dalla lex Cum quid costituiva quindi un efficace strumento giuridico per una tale concezione, e infatti non sfuggì all'attenzione dei glossatori: le glosse a tal proposito furono il germe della discussione sui debiti pecuniari, in primis il prestito di monete ${ }^{100}$. Estendendo la lex Cum quid alla moneta, i glossatori stabilirono che il requisito di D.12.1.3 dell'eiusdem generis potesse ritenersi soddisfatto solo restituendo monete dello stesso metallo, mentre che l'eadem bonitate fosse rappresentata dal medesimo valore intrinseco.

Si può dunque ben capire la forza argomentativa di un simile principio giuridico a sostegno della tesi del tempus contractus: il debitore non può che restituire monete della medesima qualità di quelle originariamente date o pattuite. L'utilizzo della lex Cum quid in tema monetario non compare solo nei nostri Brocarda, ma è presente già nella cosiddetta quaestio Quidam Creditor Lucenses di Pillio da Medicina ${ }^{101}$.

\subsection{La lex Vinum}

Per quanto riguarda invece il principio del tempus solutionis, qui la sedes materiae allegata nel brocardo è un passo di Giuliano, la cosiddetta lex Vinum (D. 12.1.22), anche in questo caso già utilizzata in tema monetario dai glossatori, e precisamente nella quaestio di Bulgaro già menzionata $^{102}$. Il passaggio si riferisce, nel caso di un mutuum di vino, all'individuazione del momento temporale su cui basare la aestimatio della controprestazione inadempiuta dal convenuto, per poter procedere alla condemnatio pecuniaria. In particolare, ci si chiede se il riferimento debba essere al tempo della restituzione, a quello della litis contestatio o infine al quello del giudicato. Giuliano risponde che, se non vi è pattuizione espressa delle parti, la fissazione del valore va riferita al momento della litis contestatio: il che, nell'interpretazione dei glossatori, corrisponde al principio del tempus solutionis inspicitur. Da qui, la ricorrente citazione che essi ne fanno in casi analoghi a

\footnotetext{
${ }^{98}$ v. Grossi, op. cit. (supra, n. 3), p. 357.

${ }^{99}$ Ivi, p. 362.

100 Taeuber, op. cit. (supra, n. 3), p. 124-125.

101 v. supra, n. 25.

102 v. supra, n. 23.
} 
quelli visti per sostenere l'opposta tesi della rilevanza del momento finale dell'obbligazione nella determinazione dei quantum.

Anche per la lex Vinum sono applicabili considerazioni analoghe a quelle svolte in precedenza: siamo in presenza di una norma che riguarda, nella sua applicazione originaria, una res fungibile completamente diversa dalla moneta, quale il vino. Anche in questo caso ai fini della comprensione dell'utilizzo di D. 12.1.22 nel caso di specie bisogna tenere conto della peculiare concezione monetaria dei glossatori.

\section{La solutio}

Dopo aver esposto nel brocardo gli opposti argomenti e le rispettive allegazioni, Azzone procede a fornire la propria solutio, che si è visto essere il contributo realmente originale del giurista bolognese ai Brocardica di Ottone. Anche per quanto riguarda la materia di nostro interesse, è la solutio a costituire il punto di svolta realmente innovativo del brocardo: se la struttura letteraria del genere si era limitata fino a quel momento a una raccolta sistematica di antinomie in funzione dialettica, con la loro risoluzione siamo dinanzi all'affermazione di un principio generale del diritto pecuniario medievale, che nell'elaborazione dei giuristi successivi fungerà da punto di partenza acquisito.

Azzone nella sua solutio prende posizione a favore del primo argomento, quello dell'eadem mensura. Esaminiamo dunque quali sono i principali punti del ragionamento del glossatore. Innanzitutto, a sostegno della sua decisione, egli utilizza un passo del Codex già contenuto nelle allegazioni, C. 1.14.7, per affermare l'irretroattività delle modifiche alle misure contenute negli statuta rispetto agli effetti sui contratti già stipulati. Si tratta anche in questo caso di una posizione già sostenuta da Pillio nella sua quaestio 79, in cui il glossatore elaborava la quaestio di Bulgaro già menzionata ${ }^{103}$, nonché ripresa dallo stesso Azzone in una sua quaestio ${ }^{104}$. Anche Azzone dunque come Bulgaro e Pillio - prende posizione in continuità con i casi esaminati, e quindi (solitamente) nell'interesse del creditore, essendo il caso più frequente nella prassi quello dell'indebolimento ${ }^{105}$. Successivamente egli esclude la rilevanza in quella sede delle allegazioni - e come si è visto sono

\footnotetext{
${ }^{103}$ Pillio, Quaestiones Sabbatinae (supra, n. 25), p. 146: 'In primis civitatis statutum ad futuros dumptaxat contractus debet pertinere, non autem ad pristinos revocari, ut C. de legibus l. Leges'. v. anche Grossi, op. cit. (supra, n. 3), p. 95. 104 v. infra, n. 106.

105 cfr. Taeuber, op. cit. (supra, n. 3), p. 229.
} 
numerose - relative alla materia successoria, in quanto la loro applicabilità è subordinata alla circostanza che si riferiscono alla volontà testamentaria del de cuius.

L'ultimo punto del ragionamento del legista riguarda il tentativo di risoluzione dell'apparente antinomia tra la lex Cum quid e la lex Vinum, neutralizzando così la principale allegazione a sostegno dell'argomento della superveniens mensura. Lo fa nella forma di una risposta alle potenziali obiezioni al riguardo: 'Nec obstat, quod legitur in ff. de rebus creditis l. Vinum, quia ibi non loquitur in alia mensura, sed in eadem'. Il fatto che egli menzioni espressamente le eventuali obiezioni possibili, è un indizio significativo di come le dispute tra legisti sul tempo dell'individuazione non dovessero essere affatto sopite ${ }^{106}$. Il giurista, dunque, prova a negare la contraddittorietà tra le due leges e a fornirne una corretta interpretazione sistematica: egli distingue la lex Vinum dalla lex Cum quid per il fatto che nel primo caso non vi è stata alcuna alterazione della misura del bene nel tempo, e dunque essa si riferisce necessariamente a una fattispecie differente rispetto a D. 12.1.3. L'applicazione del principio del tempus solutionis è dunque limitata al solo caso di aestimatio rei.

\section{La portata del brocardo}

Si è già evidenziata per alcuni aspetti la continuità del brocardo di Azzone con la tradizione giuridica precedente in tema monetario, e in particolare i punti di contatti con le quaestiones di Bulgaro e Pillio. Un'influenza che è testimoniata anche da altri lavori del nostro giurista: Annalisa Belloni, ad esempio, ha notato che le quaestiones dei due autori appena menzionate sono parallele a una quaestio contenuta all'interno di una raccolta attribuita ad Azzone e rimasta fuori dalla tradizione ufficiale, a noi pervenuta tramite un solo manoscritto ${ }^{107}$; ciò a dimostrazione del fatto che Azzone ben conosceva l'argomento e non era nuovo alla trattazione del tema della mutazione delle misure nel tempo. Che d'altra parte il glossatore non fosse estraneo alle questioni monetarie ce lo testimonia

\footnotetext{
${ }^{106}$ Ivi, p. 230.

${ }^{107}$ È il ms. Léon, Biblioteca de la Colegiata de S. Isidoro, 16, in cui le questioni di Azzone precedono una raccolta di testi proprio di Pillio. Tali questioni sono state edite da Belloni, op. cit. (supra, n. 23), p. 123-172. Sulla raccolta v. ivi, p. 36, 48, 125-126; per la quaestio di nostro interesse (la $\left.\mathrm{n}^{\circ} 10\right) \mathrm{v}$. invece ivi, p. 143-144, e per una comparazione testuale delle quaestiones parallele $i v i$, p. 185-186. Basti qui notare che, riportato è il medesimo, la trattazione fatta da Azzone differisce da quella di Pillio, e non solo stilisticamente: pochi sono gli argomenti comuni addotti, e anche i passi del Corpus iuris civilis citati differiscono notevolmente. In ogni caso, Azzone riporta sulla scorta di Pillio, seppur solo implicitamente, il principio della irretroattività della legge, citando come primo argomento a favore del creditore C 1.14.7: una allegazione che - come si è visto - compare anche nel nostro brocardo e specialmente nella solutio. In questa quaestio, infine, anche il giurista bolognese adotta la medesima posizione di Pillio e Bulgaro, pur senza citare i suoi predecessori.
} 
anche una sua glossa a D. 18.1.1 (la lex Origo emendi), in cui il legista afferma apertamente la rilevanza del contenuto metallico della moneta, sancendo la concezione della moneta-merce ${ }^{108}$. Anche Azzone infine, come i glossatori suoi predecessori, afferma il venire in considerazione del momento iniziale del contratto e opera una riconduzione del problema giuridico dell'alterazione monetaria a una questione di interpretazione contrattuale.

Non mancano tuttavia differenze e le innovazioni significative introdotte dal brocardo. Innanzitutto, il principio giuridico qui affermato non si limita ad esaminare separatamente - come ad esempio avveniva in Pillio - il fenomeno dell'alterazione statutaria delle unità di misura e quello della mutatio monetae, ma sviluppa un argomento generale, valido per le variazioni statutarie di tutte le misure, che si tratti sia di moneta sia di altro. I due casi (quello delle misure e quello delle monete) sono dunque affrontati e risolti in maniera uniforme, fornendo una regola giuridica di applicabilità generale, senza distinzioni tra diminuzioni e ingrandimenti della misura ${ }^{109}$.

Contrariamente alla quaestio di Pillio, poi, il brocardo di Azzone non limita il proprio campo di applicazione alla semplice datio, termine che presuppone ci si stia riferendo a un negozio di mutuum (e quindi al prestito di moneta), ma si riferisce genericamente a un contractus; è dunque applicabile a tutte le fattispecie contrattuali indipendentemente dalla causa. Mentre i glossatori fino ad allora si erano limitati ad esaminare il problema monetario rispetto al caso ristretto del mutuum affermando il principio del tempus dationis - la portata del brocardo azzoniano va ben oltre, riferendosi al momento in cui il contratto è stato concluso, ossia al tempus contractus. Il brocardo di Azzone, liberatosi definitivamente della datio, si applica senza alcuna ambiguità a tutti i tipi contrattuali, compresi quelli che si perfezionano in virtù del semplice consenso ${ }^{110}$.

Il brocardo di Azzone era quindi, in pratica, una singola risposta da adattare a tutti i casi, riassunta nella formula 'eadem mensura vel moneta debetur, que erat tempore contractus' ${ }^{111}$. In virtù di questa generale applicabilità, la regola giuridica del tempus contractus contenuta nel brocardo di Azzone rappresenta non un caso specifico, ma la formulazione - attraverso la solutio - di un principio per una teoria monetaria più generale, riassumibile nella tutela della volontà originaria delle parti in

\footnotetext{
108 Glossa di Azzone a 'dominiumque', contenuta in Ms. Bamberg StaatsBibliothek, iur. 11, fol. 209r e riportata in Taeuber, op. cit. (supra, n. 3), p. 333, n. 923. Sull'argomento v. in generale ivi, p. 333; Ernst, The Glossators' (supra, n. 3), p. 226; Sargent, Velde, op. cit. (supra, n. 1), p. 94.

${ }^{109}$ Come evidenziato da Ernst, The Glossators' (supra, n. 3), p. 235.

${ }^{110}$ Ivi, p. 235-237.

111 Sargent, Velde, op. cit. (supra, n. 1), p. 75.
} 
sede di interpretazione del contratto ${ }^{112}$. La questione del debito pecuniario fu risolta dunque come un caso di interpretazione contrattuale, il che rese possibile darne un'interpretazione metallista e non nominalistica, tutelando così i diritti delle parti private contro i pregiudizi arrecati dalle disposizioni pubbliche. Come principio di interpretazione contrattuale, allora, il brocardo aveva necessariamente portata generale, applicandosi a tutti i contratti e a fortiori alle obbligazioni pecuniarie ${ }^{113}$. Non solo: la riconduzione del problema della mutazione monetaria nel perimetro dell'interpretazione contrattuale, ha il vantaggio di lasciare alle parti la libertà di poter specificare nel contratto quale sarà la moneta di riferimento ${ }^{114}$.

Azzone, insomma, con il suo brocardo indicò un modello generalizzato, che ebbe ampia accettazione tanto tra i romanisti quanto tra i canonisti ${ }^{115}$. In ambito civilistico, esso venne ripreso da Accursio, che confermò questa dottrina nella sua Magna Glossa ${ }^{116}$, consacrandone il successo tra i giuristi successivi e l'ingresso nella communis opinio. Anche la prassi tese ad adeguarsi al principio sancito da Azzone: se ne trovano chiare tracce per quanto riguarda il Comune di Bologna ${ }^{117}$. Per quanto riguarda invece i canonisti, esso influenzò grandemente il diritto della Chiesa, anche perché le soluzioni proposte erano in linea con l'ideale morale della giustizia commutativa ${ }^{118}$.

\section{Conclusioni}

Come si è avuto modo di accennare nelle prime pagine del presente studio, una dei nostri obiettivi nell'analisi del brocardo azzoniano riguarda la necessità di rivalutare l'importanza del contributo dei glossatori all'elaborazione di una sistematica giuridica in tema pecuniario. L'Europa medievale passò, nel corso di pochi secoli, dall'assenza di un'elaborazione teorica sulla moneta allo

\footnotetext{
112 cfr. Grossi, op. cit. (supra, n. 3), p. 5: 'I giuristi intermedi infatti, lungi dal limitarsi a una soluzione minuta del problema della mutatio monetae, preferiscono risolverlo immettendolo nell'àmbito dei principii generali dell'ordinamento e presupponendo quindi alle loro soluzioni questi principii'.

113 Taeuber, op. cit. (supra, n. 3), p. 231.

114 Ernst, The Glossators' (supra, n. 3), p. 237.

${ }^{115}$ Ivi, p. 77.

${ }^{116}$ Cfr. glossa a D. 12.IL1.3 (lex Cum quid), 'Bonitate', fol. 192r: 'quo ad intrinseca ut sit similis saporis. licet precium cursu temporis sit mutatum ut j. e. Vinum'; e glossa a D 12.1.22 (lex Vinum), 'Cum petitum esset', foll. 194v-195r, in Accursio, Accursii glossa in Digestum vetus, Venezia, 1488 [rist. anast. in CGJC, 7, Torino 1969].

${ }^{117}$ v. Taeuber, op. cit. (supra, n. 3), p. 243-244.

118 G. Hubrecht, Quelques observations sur l'évolution des doctrines concernant les paiments monétaires du XII eau XVIIIe siècle, in: Aequitas und bona fides: Festgabe zum 70. Geburstag von August Simonius. Hrsg. von der Juristischen Fakultät der Universität Basel, Basel 1955, p. 135.
} 
sviluppo di un fecondo pensiero giuridico sul tema. Nel corso di queste pagine, si è cercato di ricostruire i primi passi di questo sviluppo attraverso uno dei testi che maggiormente hanno influito sulla formazione della dottrina monetaria. Si è accennato alla stretta correlazione tra tre fenomeni coevi: la ripresa dell'economia e dei commerci, la conseguente monetizzazione e la nascita della cultura giuridica. La concezione regalistica e pubblicistica della moneta, che era riemersa gradualmente in Occidente, annoverava la moneta tra le regalìe, e precisamente configurava in capo al sovrano due prerogative estremamente problematiche: la mutazione del contenuto intrinseco (renovatio monetae) e la variazione del valor impositus, ossia del suo valore nominale. L'applicazione e l'abuso di queste due forme di mutazione monetaria, con i conseguenti fenomeni distorsivi che si sono esaminati, trovava sovente la contrarietà dell'emergente classe mercantile, poiché danneggiava l'economia commerciale in espansione.

A queste istanze dei gruppi sociali fu chiamato a dare una risposta proprio il diritto colto, che sin dall'inizio sembra interessarsi al problema, con le quaestiones di Bulgario e Pillio, e che trova una definitiva sistemazione nel lavoro di Azzone da noi esaminato. La successiva elaborazione dello ius civile confermò sostanzialmente l'impostazione dei primi glossatori: si venne così configurando una teoria che inquadrava la moneta come merce, e che riconduceva la risoluzione dei problemi monetari - e nello specifico dei casi in cui le mutazioni monetarie si riverberavano sull'autonomia privata - all'alveo dell'interpretazione contrattuale. La strada intrapresa dai legisti dunque fu quella di tutelare i privati e i loro rapporti pecuniari dalle indebite ingerenze e dagli squilibri causati dall'intervento del sovrano. Ciò fu realizzato negando la rilevanza del valor impositus ed esaltando il contenuto metallico della moneta, individuandone inoltre l'entità sulla base dello stretto principio del tempus obligationis. I glossatori dunque svilupparono una concezione della moneta fondamentalmente metallista, mossi da un interesse di tipo equitativo, volto a tutelare la giustizia sostanziale del rapporto giuridico ed economico, contro le distorsioni create dal potere pubblico ${ }^{119}$.

A fronte di questa svolta nel pensiero giuridico medievale, ben rappresentata nell'opera azzoniana, poco valore sembra avervi attribuito la storiografia. In primis quella economica, che, mentre ha esaltato il ruolo svolto dalla Scolastica e dalla filosofia medievale in tema monetario, vedendo nella riscoperta di Aristotele le basi per lo sviluppo di una vera elaborazione concettuale al riguardo, ha d'altro canto teso a sottovalutare l'apporto originale del pensiero giuridico al tema. Anche la storiografia di ambito giuridico sembra aver dedicato ben poca attenzione alla questione, $o$ addirittura aver unanimemente minimizzato l'apporto intellettuale del diritto colto all'idea di

\footnotetext{
${ }^{119}$ Sugli aspetti equitativi dell'elaborazione giuridica in tema monetario v. in particolare Grossi, op. cit. (supra, n. 3), passim.
} 
moneta $^{120}$. Così Grossi, che pure coglie correttamente e acutamente molte questioni, riconosce ai soli commentatori - su cui il suo lavoro si focalizza - l'elaborazione di un pensiero sensibile alla realtà sociale e politica del suo tempo ${ }^{121}$. Per quanto riguarda invece i glossatori, egli ritiene la loro produzione intellettuale sulla moneta incerta, grezza e non sistematica: 'il concetto giuridico di moneta era per loro fondamentalmente oscuro' ${ }^{122}$. Non è molto diversa la valutazione di Ernst: 'With a little exaggeration, one could say that the glossators did not have any concept of money at all. Coins themselves were demonetised ${ }^{123}$. Lo studioso tedesco considera l'elaborazione della teoria della moneta-merce non come il prodotto di un pensiero originale dei glossatori, ma come la semplice adozione giuridica di un fatto comune all'esperienza economica; per essi, insomma, il metallismo non sarebbe stata una scelta, ma una necessità ${ }^{124}$.

Ciò che va innanzitutto detto, è che la posizione dei glossatori in tema monetario non possa considerarsi la semplice e pacifica recezione, a livello giuridico, della realtà economica da essi vissuta. Certamente l'idea della moneta come equivalente del suo contenuto intrinseco non è un'esclusiva dei glossatori, ed è innegabile che il suo sviluppo nasca dalla prassi alto-medioevale, diretta conseguenza della scarsa monetizzazione dell'economia e dell'assenza di fiduciarietà nella circolazione monetaria. Nonostante ciò, sarebbe errato e semplicistico attribuire al metallismo medioevale un carattere monolitico e una indiscussa continuità che va da Isidoro di Siviglia ad Accursio, recepito dai glossatori meramente ed automaticamente in ragione della prassi ${ }^{125}$. Al contrario, la teoria della moneta-merce sviluppata dai glossatori è una scelta consapevole e coerente, motivata da precise esigenze giuridiche e sociali. Ne è la dimostrazione il fatto che la sua formulazione ed accettazione sembra essere stata tutt'altro che pacifica in dottrina: una considerazione a cui sembra spingerci la stessa analisi dei generi letterari - fortemente dialettici attraverso cui l'elaborazione si è sviluppata, vale a dire le quaestiones e i brocarda, segni di un dibattito giuridico che deve essere stato decisamente vivace e aperto fino almeno ad Azzone ${ }^{126}$. E il

\footnotetext{
${ }^{120}$ v. ad esempio Salvioli, op. cit. (supra, n. 3), p. 73: 'Prima di Bartolo, nelle Glosse e nei Commentarii al diritto romano, manca una seria e profonda trattazione della moneta, e tutto si restringe a pochi cenni intorno al falso nummario. Anche le questioni che scaturiscono in causa dell'alterazione delle monete sono insufficientemente sviluppate'.

${ }^{121}$ Grossi, op. cit. (supra, n. 3), p. 335.

${ }^{122}$ Ivi, p. 137.

${ }^{123}$ Ernst, The Glossators' (supra, n. 3), p. 244.

${ }^{124} \mathrm{Ivi}$, p. 245.

${ }^{125}$ In questo senso si esprime invece Grossi, op. cit. (supra, n. 3), p. 184,

${ }^{126}$ v. supra, n. 28; Taeuber, op. cit. (supra, n. 3), p. 230. Anche successivamente non mancarono di certo i tentativi di ritagliare uno spazio all'iniziativa del potere pubblico rispetto alla sfera di autonomia dei privati. Lo stesso brocardo di Azzone, come si è visto, si applica a tutti i casi di debito derivante dal contratto, lasciando dunque espressamente escluso
} 
nostro stesso brocardo, con la sua struttura antitetica e inizialmente sprovvisto di solutiones, ne costituisce un segnale evidente, soprattutto se si considerano le origini e la funzione del genere e la necessità, soddisfatta per mano di Azzone stesso, di approntare soluzioni di ordine sistematico che risolvessero le antinomie.

In secondo luogo, va notato come l'impostazione dottrinaria dei glossatori sul punto, di cui il brocardo di Azzone è una rappresentazione plastica e un punto fermo, non è fondata sul mero riguardo al contenuto intrinseco del bene, bensì è mossa in primis dall'esigenza di tutela della volontà originaria delle parti all'interno di un rapporto contrattuale. Sebbene sia vero che la riduzione del problema monetario a un caso di interpretazione contrattuale impedisce lo sviluppo di un'elaborazione giuridica che riconosca la specificità della moneta rispetto alle altre es $^{127}$, ciò non è il frutto di una semplificazione o di una incomprensione da parte dei giuristi, bensì di una precisa scelta teleologicamente orientata alla tutela delle parti contrattuali. I glossatori alle prese con la riscoperta dei testi giustinianei avrebbero potuto configurare la moneta alla luce della enorme varietà di riferimenti normativi sull'argomento; scelsero invece, in piena consapevolezza, di applicare ad essi la disciplina comune ai beni fungibili. Solo in tale maniera, infatti, ossia negando scientemente alla moneta una specificità sua propria, era possibile realizzare l'obiettivo che i doctores si erano prefissi: la tutela dei contraenti e, tramite essa, la tutela di un'intera classe sociale, il ceto mercantile emergente, vero protagonista del XII e XIII secolo, i cui interessi creditori erano minacciati dalle arbitrarie mutazioni delle unità di misura da parte del potere pubblico, che causava un'instabilità monetaria nociva per gli scambi. Siamo dinanzi, dunque, a una presa di posizione cosciente e perfettamente sensibile alle istanze politiche, sociali ed economiche del tempo.

Altrettanto semplicistico sarebbe negare ai primi prodotti del diritto colto in tema monetario qualunque portata generale, relegandoli alla semplice trattazione casistica. Vero è, come si è da più

il caso di obbligazioni pecuniarie derivanti da fonti diverse (v. Hubrecht, op. cit. (supra, n. 118), p. 135). Così ad esempio i giuristi medievali, pur tutelando l'autonomia privata nelle fattispecie contrattuali, riconobbero le prerogative pubbliche nell'ipotesi di atti di imperio: è il caso della distinzione - tutt'altro che pacificamente accettata - tra debitum ex lege e debitum ex contractu operata da Guillaume Durand (c, 1230 - 1296), secondo il quale nel caso di obbligazioni pecuniarie fissate da un atto pubblico il pagamento dovesse avvenire secondo la moneta corrente: v. Guillaume Durand, Speculi pars quarta, Lyon, 1541, lib. IV, p. III, De obligationibus et solutionibus, § Nunc aliqua, $\mathrm{n}^{\circ}$ 9, fol. 136r; Grossi, op. cit. (supra, n. 3), p. 228, Questa dottrina aprì in epoca più tarda la strada a posizioni fortemente statalistiche, come quella del canonista Jean Favre (m. dopo il 1340), vicino al re Filippo il Bello, che considerò la materia monetaria prerogativa esclusiva del monarca: Jean Favre, In justiniani codicem breviarium, Lyon, 1550, p. 216; Sargent, Velde, op. cit. (supra, n. 1), p. 8990; Hubrecht, op. cit. (supra, n. 118), p. 140; Ernst. The Legists' (supra, n. 21), p. 131.

127 Come nota Ernst, The Glossators' (supra, n. 3), p. 238. 
parti osservato, che ad essi manca una teoria uniforme e astratta della moneta ${ }^{128}$, tuttavia non vi si può vedere neanche soltanto la sommatoria di casi concreti. La portata generale e sistematica di un pensiero monetario coerente è proprio il principale traguardo del brocardo di Azzone: una considerazione che si può trarre sia alla luce della funzione stessa dei brocarda come genere letterario, sia dall'analisi del testo concreto. Il giurista bolognese, infatti, sembra ben consapevole della necessità di ricondurre le decisioni di diritto romano in tema monetario ad un unico principio giuridico, generalmente applicabile a tutte le fattispecie contrattuali. E se esso non costituisce un punto di arrivo in questo senso, che comunque avrà uno sviluppo nella dottrina dei commentatori, è certamente un momento di svolta nell'affermazione di questo principio.

Per concludere si può dire dunque che nell'ambito dello ius civile non sembra di potersi ridurre l'elaborazione dei primi glossatori, e di Azzone in particolare, a meri passi incerti, prodromici al Commento e al diritto comune: essi con un'operazione ermeneutica e selettiva dei testi giustinianei, hanno costruito i primi elementi di una teoria giuridica che verrà perfezionata nel secolo successivo, nella piena consapevolezza maturata nell'alveo di un intenso dibattito dottrinario ${ }^{129}$. L'elaborazione monetaria della Glossa costituisce a tutti gli effetti il punto di partenza della storia dogmatica moderna sul tema ${ }^{130}$.

\footnotetext{
${ }^{128}$ Ivi, p. 244; Taeuber, op. cit. (supra, n. 3), p. 331-339.
}

129 v. supra, nn. 28 e 126. Le posizioni minoritarie rispetto alla dottrina metallistica, che esaltavano le caratteristiche estrinseche della moneta, non mancarono neppure successivamente, ma si affermarono compiutamente solo nel corso del XVI secolo (Grossi, op. cit. (supra, n. 3), p. 323). Le si trovano ad esempio in Ugolino de' Presbiteri (m. c. 1233) (v. Taeuber, op. cit. (supra, n. 3), p. 163; Ernst, The Glossators' (supra, n. 3), p. 228) e Odofredo Denari (m. 1265) (v. Ernst. The Legists' (supra, n. 21), p. 135); oppure - al tempo dei commentatori - in Pierre de Belleperche (c. 1230 - 1308) e Iacopo d'Arena (m. 1296), i quali considerarono la moneta in termini diversi dal suo conenuto intrinseco: v. Commentario a Inst. 4.6.16 ('Sequens'), in: Pierre de Belleperche, In libros Institutionum Divi Iustiniani commentarii, Lyon, 1536 (rist. anast. in Id., Lectura Institutionum, [Opera iuridica rariora, 7,] Bologna, 1972), p. 690; Taeuber, op. cit. (supra, n. 3), p. 197-198; Sargent, Velde, op. cit. (supra, n. 1), p. 79-80; Ernst. The Legists' (supra, n. 21), p. 129.

${ }^{130}$ Come nota Ascarelli, La moneta (supra, n. 96), p. 5. 
Sigle dei manoscritti:

AV Avignon, Bibliothèque du Musée Calvet, 762, fol. 59v-60r

BAM1 Bamberg, StaatsBibliothek, Jur. 27, fol. 10v-11r (p. 20-21)

BAM2 Bamberg, StaatsBibliothek, Jur. 34, fol. 58v-59r

BO Bologna, Biblioteca Albornoziana del Collegio di Spagna, 118, fol. 185v

BRE Bremen, Staats- und Universitätsbibliothek, a 131, fol. 211v

BRU Bruxelles, Bibliothèque Royale Albert Ier, 131-134, fol. 7r

CA1 Cambridge, Gonville and Caius College, 665/261, fol. 130v

CA2 Cambridge, Pembroke College, 139, fol. 120v

CH1 Vaticano, BAV, Chigi E.VII.211, fol. 37r

CH2 Vaticano, BAV, Chigi.E.VII 218, fol. 5v

CH3 Vaticano, BAV, Chigi E.VII.218, fol. 42r

DU Durham, Cathedral Library, C.III.12, fol. 227r

LA Laon, Bibliothèque Municipale, 395, fol. 20v

LE1 Leipzig, Universitätsbibliothek, 992, fol. 76rv

LE2 Leipzig, Universitätsbibliothek, 992, fol. 100r

MA Madrid, Biblioteca Nacional, 676, fol. 121v

MI Milano, Biblioteca Ambrosiana, E.23 inf., fol. 76v

MU1 München, Bayerische Staatsbibliothek, clm. 213, fol. 73r

MZ Mainz, Wissenschaftliche Stadtbibliothek, I. 502, fol. 2r

O1 Vaticano, BAV, Ott. Lat. 1298, fol. 6v

O2 Vaticano, BAV, Ott. Lat. 1299, fol. 64v

P1 Paris, Bibliothèque Nationale, lat. 4601, fol. 5r

P2: $\quad$ Paris, Bibliothèque Nationale, lat. 4601, fol. 26r

P3 Paris, Bibliothèque Nationale, lat. 4609, fol. 9v 
R Vaticano, BAV, Reg. lat. 1126, fol. 92r

SI Sion, Archives de l'État du Valais, 83, fol. 296v.

TO Torino, Biblioteca Nazionale Universitaria, K.I.19, fol. 46rv

TR Troyes, Médiathèque du Grand Troyes, 1751, fol. 80v

VA1 Vaticano, BAV, Vat. lat. 2343, fol. 95v

VA2 Vaticano, BAV, Vat. lat. 7778, fol. 65r

W1 Wien, Österreichische Nationalbibliothek, Cvpl. 2088, fol. 9v-10r

W2 Wien, Österreichische Nationalbibliothek, Cvpl. 2091, fol. 9v

WO Worcester, Cathedral Library, F. 14, fol. 114rv

Non ho potuto consultare personalmente i seguenti manoscritti:

LM Lambach, Stiftsbibliothek, chart. 160

ME Metz, Bibliothèques-Médiathèques de la Ville de Metz, 102

MU2 München, Bayerische Staatsbibliothek, clm. 3507

SE La Seu d'Urgell, Arxiu Capitular d'Urgell, 2022

TL Toledo, Biblioteca de la Catedral, 39-28

TS Tours, Bibliothèque municipale, 657 
L'edizione

1. Il brocardo

$<1$. $>$ Eadem mensura vel moneta debetur, que erat tempore contractus.

<2.> In Decreto I q. I Principatus (c. 25 C. I q. 1).

$<3$. In Authentico Ut cum de appellatione cognoscitur (Nov. 115) § I.

$<4$. $>$ ff. si quis cautionibus Si quis quam (D. 2.11.11) et 1 . Qui autem $\S$ illud (D. 2.11.12.1).

$<5$. $>$ ff. de edendo Ubi (D. 2.13.8) § I.

$<6 .>$ C. de legibus et constitutionibus Leges et constitutiones (C. 1.14.7).

$<1$.> Eadem ... vel] quod vere $R$ TO debetur om. $C H 1$ que ... contractus om. Ol W2 que tempore contractus erat $B O B R U C A 2 C H 1 C H 3 M I M Z W O$ que tempore contractus erant $\mathrm{CH} 2$ que tempore contractus fuit $P 1$

<2.> In ... principatus om. BRU CH1 CH2 MI O1 O2 P1 W1 W2 WO I ${ }^{\mathbf{1}} \mathrm{om}$. CA1 TR DU q. I] Rubrica I $C H 3$ causa agg. nell'interlineo $D U$

<3.> In om. P1 ut om. CA2 CH1 CH2 MI TR WO et $B R U$ ut cum om. O2 de om. $D U$ cognoscitur] cognoscitur cum $W 1$

$<4$.> ff. ... illud om. W2 ff.] in Authentico $L A M A R \quad$ Si] ut $R$ quis om. WO quid MI quam Nella Vulgata del Digesto compare quempiam (quendam nell'ed. critica di Mommsen), che però non compare in nessuno dei manoscritti. om. MI quem AV BAM1 BAM2 BO BRU P3 VA2 quam corr. quamquam $\mathrm{CHI}^{2}$ quamquam $\mathrm{CH} 3 \mathrm{MZ} \mathrm{P1} \mathrm{Wl}$ quemquam $\mathrm{CH} 2 \mathrm{O} 2 \mathrm{WO}$ et l. om. $\mathrm{CH} 1$ et ... illud om. BRU CH2 CH3 MI MU1 MZ O1 O2 P1 W1 Qui ... illud add. CH1 ${ }^{2}$ autem] ante AV BAM1 BAM2 BO BRE CA1 CA2 SI P3 VA1 VA2

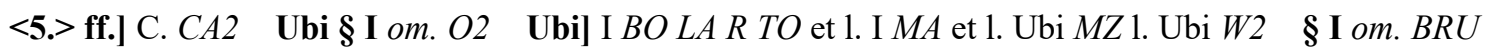
CH2 CH3 MI MZ O1 P1 W1 W2 WO add. $\mathrm{CH}^{2}$

<6.> C. ... constitutiones om. BAM1 BAM2 BRE BRU CH1 CH2 CH3 MI MZ O1 O2 P1 SI VA1 VA2 W1

$W 2 W O \quad$ C.] ff. $D U$ legibus] ligibus $D U$ 
$<7 .>$ ff. si certum petatur (D. 12.1) 1. III, in fine.

$<8$. $>$ ff. de evictionibus Bonitatis (D. 21.2.13).

$<9$. $>$ ff. de legatis II Lucius Titius (D. 31.1.22).

$<10$. $>$ ff. de legatis III Nomen debitoris $\S$ I (D. 32.1.34.1).

$<11$. $>$ ff. de legatis III 1. Uxorem et filium $\S$ testamento (32.1.41.4).

$<12$. $>$ Item constitutiones formam dant futuris negotiis.

$<13$. $>$ In Decreto, D. XXXI, ante triennium (d. 31 c. 1).

$<$ 14. $>$ ff. de iudiciis 1. Si quis postea $<$ quam $>$ (D. 5.1.7).

$<$ 7. $>$ ff. ... fine $o m$. $W 2$ ff.] ut ff. $O 2$ petatur I. III in fine] 1. III in fine petatur $T R \quad$ l. ... fine] in fine 1. III SI III ] II MZ in fine om. BRU CH1 CH2 MI O1 O2 P1 WI WO

$<$ 8. $>$ de evictionibus] devictionibus $D U$ Bonitatis] ne istis $M I$ ne ist. con segno abbreviativo sulla $\mathrm{CH}$ nec inst. con segno abbreviativo sulla t $C H 3$ ne inst. $B R U$ ne inst. con segno abbreviativo sulla $\mathrm{t} M Z$ non inst. $W O$

$<$ 9.> ff. ... Titius om. CH3 $\quad$ II] I $B O M A R$ TO WO III $M I$

<10.> ff. ... § I om. AV BAM1 BAM2 BO BRE CA1 CA2 DU LA MA MU1 P3 R SI TO TR VA1 VA2 W2

ff. ... III] et 1. MI III] II $C H 3$ iudi. $O 2 W 1$ Nomen ... I] $\S$ I nomen debitoris $P 1 \quad$ Nomen] in nominibus $M I$ non $O 1$ debitoris] debet $C H 1 O 1$ WI WO debitum $C H 3$ om. $M Z$ I] $†$ OI

$<$ 11.> ff. ... testamento om. W2 ff. de legatis III] et $A V B A M 1$ BAM2 BO BRE CA1 CA2 DU MA MU1 P3 SI TO TR VA1 VA2 ff. de legatis III 1.] et 1. III LA de legatis III l. om. CH3 eodem CH1 CH2 MI $M Z O 1$ WO de $B R U P 1$ 1. om. O2 WI filium ] filii CHI CH2 CH3 MI MZ O1 WO 1. Filii BRU P1 1. Filium AV BAMI BAM2 BO BRE CAI DU LA MA MU1 P3 R SI TO TR VAI VA2 testamento] testator AV BAMI BAM2 BO BRE CA1 DU LA MA MU1 O2 P3 R SI TO TR VA1 VA2 testor W1 testamento non aliter CH2 CH3 OI P1 testamento naturaliter $B R U M Z W O$ testamento natura $M I$ testamento $\dagger C H 1$

<12.> Item ... negotiis om. $\mathrm{BRU} \mathrm{CH} 1 \mathrm{CH} 2 \mathrm{CH} 3 \mathrm{MI} M \mathrm{MZ} O \mathrm{O} 2 \mathrm{Pl}$ W1 W2 WO constitutiones] constitutionis $V A 1$ formam dant futuris negotiis] dant futuris negotiis formam $A V$ dant] habeant $L A$ MA R dat MUI SI TO VAI datum BAMI

<13.> In ... triennium om. BRU CH1 CH2 CH3 MI MZ O1 O2 P1 W1 W2 WO Decreto] Decretis MU1 D. XXXI] XXXI D. $C A 2$ D.] *** VA1 ante] autem BAM2 $M A$

$<\mathbf{1 4}$.> ff. ... postea $o m$. $W 2$ iudiciis] iudicis $B A M 1$ indiciis $V A 2 \quad$ l. om. CH1 MU1 Si quis] Si quis $B R U$ 
$<15$. $>$ C. de pascuis publicis 1. I (D. 11.61.1).

$<16$. $>$ C. de prediis et omni $<$ bus $>$ rebus naviculariorum (D. 11.3) 1. II.

$<17$. $>$ Supra, initium spectandum, tempus contractus debere inspici.

$<18$. $>$ Supra, Repetita die, expresse.

$<19$. $>$ Infra, de pactis, Rubrica I et ultima.

$<20$. $>$ Infra, de emphiteota, Rubrica II.

$<21$. $>$ Nota, quedam emuntur, que loco venditorum supponuntur.

$<22$. $>$ Superveniens debetur, sive maior sive minor sit.

<15.> C. ... II om. AV BAM1 BAM2 BO BRE CA1 CA2 DU LA MA MU1 P3 R SI TO TR VA1 VA2 W2 C.] ff. $B R U C H 1 C H 3 M I M Z O 2 W 1 W O$ pascuis] pastu. $C H 1$ pascuiis $O 2$ publicis] et p. $O 1$ p. $c o n$ segno abbreviativo sovrascritto $O 2 W 1$ privatis $C H 3 \mathrm{MZ}$ pec con segno abbreviativo -us sulla c. CH1 I] II BRU CH1 CH2 MI OI O2 P1 W1 III WO

<16.> C.... II om. O1 W2 C.] C. ff. $A V$ omni] communi $P 1$ communi esp. omni $C A 2$ rebus om. VA1 naviculariorum] ma. AV BAM1 BAM2 BO BRE CA1 CA2 DU LA MA MU1 MZ P3 R TO TR VA1 VA2 manu. $C H 3$ nota $M I$ nata $B R U C H 1$ WO

<17.> Supra ... inspici om. BRU CH1 CH2 CH3 MI MZ O1 P1 W2 WO Supra om. W1 Si $R$ initium] initius $V A 1$ tempus] tempore $C A 2 O 2 R$ et tempore $T R$ et que tempus $D U$

<18. S Supra ... etc om. BRU CH1 CH2 CH3 MI MZ OI P1 R VA2 W2 WO Repetita] de repetita AVBO CA1 CA2 DU MA MU1 TR et repetita BAM1

<19.> Infra ... ultima om. BRU CH1 CH2 CH3 LA MA MI MZ OI P1 R W2 WO pactis] act. pactis con segno abbreviativo sulla $\mathrm{t}$. CA1 I] a TO et om. DUVA1

<20.> Infra ... 2 om. BRU CH1 CH2 CH3 MI MZ O1 P1 P3 W2 WO de emphiteota] demphiteota $D U$ <21.> Nota ... supponuntur om. BRU CH1 CH2 CH3 MI MZ O1 P1 W2 WO Nota] ex nota $R$ quedam] quod que $W 1$ emuntur] emunet. $T O$ que om. $O 2$

$<$ 22. $>$ Superveniens ... sit] $\dagger$ maior sive minor sit superveniens debetur $P 3$ Superveniens] Econtra quia superveniems $M I$ Infra de superveniens $V A 2$ sive maior sive minor] sive minor sive maior $B O \quad$ sive $^{1}$ om. MI sine VA2 sive $\left.{ }^{2}\right]$ sine DUVA2 sive minor] minorve BRU CH1 O1 O2 P1 W1 W2 WO et minorve CH2 sit om. BAM1 TO VA2 
$<23$. $>$ In Decreto XXIII, q. IIII Si Ecclesia (C. 23. q. 4 c. 42).

$<24$. $>$ ff. de conditionibus et demonstrationibus Filie (D. 35.1.28) § ultimo.

$<25$. $>$ ff. de liberis et posthumis Placet (D. 28.2.4).

$<26$. $>$ ff. de legatis II Imperator (D. 31.1.70), § ultimo.

$<27$. $>$ ff. si certum petatur Vinum (D. 12.1.22).

$<28$. $>$ ff. de pignoribus Cum tabernam (D. 20.1.34), rubrica I.

$<29$. $>$ ff. de furtis Infitiando $\S$ infans (D. 47.2.68.2).

$<30$. $>$ ff. de fideiussoribus Si quis (D. 46.1.56) § si nummos.

$<31$. $>$ ff. de operis libertorum (D. 38.1) 1. I.

<23.> In ... Ecclesia om. BRU CH1 CH2 MI O1 O2 P1 W1 W2 WO Decreto Decretum $B R E \quad$ XXIII] XXIIII $V A 2$ XXXIII $R$ XXX $L A$ *** TO $\quad$ IIII] III MA MU1 IIIor BAM1 $\quad$ Si om. VA2 Ecclesia] econtra AV BO BRE CH3 DU LA SI TO VAI

$<\mathbf{2 4}$.> ff. ... ultimo om. VA2 $\quad$ ff.] Supra $W 1 \quad$ demonstrationibus] *** TO Filie] de filie $\mathrm{CH}^{*}$ § ultimo om. BRU CH1 CH2 MI MZ O1 P1 WO ultimol I CH3 O2 WI

Seguono i paragrafi: $<24.1>$ ff. qui satisdare cogantur De die ponenda (D. 2.8.8) $<24.2>$ ff. de contrahenda emptione et venditione Si in emptione (D. 18.1.34) Rubrica I $P 3$

$<\mathbf{2 5}$.> ff. ... placet om. O1 SI W2 Placet] Hii placet $B R U C H 1$ CH2 MI O2 W1 WO

<26.> ff. ... ultimo om. SI W2 $\quad$ II] III $M I O 2 W 1$ 1. II $R \quad$ Imperator] Imperatorum $W O$

$<27 .>$ ff. ... Vinum om. BRU MI Vinum] Domum $B O T O$

$<28$.> ff. ... I om. $B O \quad$ Cum] 1. Cum $O 2 W 1$ quod $M I$ tabernam] taberna $R S I V A 1$ rubrica I] priorum CH3 I rubrica CA2 om. BRU CH1 CH2 MI MZ O1 O2 P1 W1 W2 WO

Segue il paragrafo: $<28.1>$ C. de usuris 1. penultima (C. 4.32.27) $O 2 \mathrm{Wl}$

<29.> ff. ... infans om. BRU CHI MI WO Infitiando om. O2 W1 inficiendo LA MA PI R SI TO VAI infaciendo $D U$ §om. SIVAI infans ] in suus $P 1$

$<$ 30. $>$ ff. ... nummos om. $W 2$ fideiussoribus] fidei commissis $W O$ si quis] con. si quis $B R U C H 1 C H 2$ $O 1 O 2 \mathrm{Wl}$ contra si quis $P 1$ quis] quid $A V B O C A 1$ CA2 DU MA MU1 R TO TR si nummos] infans CHI MI infans si nummos $B R U W O \quad$ si om. SI nummos] inimicos $O 1$

$<31$. $>$ ff. ... I om. W2 $\quad$ l. om. $\mathrm{CH} 2$ 
$<32$. $>$ ff. de verborum obligationibus Inter stipulantem (D. 45.1.83) $§$ ultimo.

$<33$. $>$ ff. quorum legatorum Diversum (D. 43.3.2).

$<34$. $>$ Eadem mensura vel moneta debetur, etc. $<35$. $>$ Solutio. Eadem mensura debet prestari, et eadem moneta: vel, si alia prestetur, habeatur ratio dampni. $<36$. $>$ Statuta post contractum facta, futuris contractibus dant formam, non preteritis, ut C. de legibus et constitutionibus Leges (C. 1.14.7). <37.> Nec obstat quod legitur ff. de legatis II Imperator $\S$ ultimo, et de pignoribus 1. Cum tabernam, et de furtis Infitiando $\S$ infans (D. 47.2.78.2), <38.> quia in his legibus non prohibetur, quin possint peti ea, que tempore

<32.> ff. ... ultimo om. $W 2$ ff. In $B R U$ Inter] iur con segno abbreviativo soprascritto VA2 Inter stipulantem] insti. $\mathrm{CH} 3 \quad$ stipulantem] pap. con segno abbreviativo soprascritto $O 1 \quad \S$ ultimo om. $R$ $<33$. $>$ ff. ... diversum $o m$. $W 2$ legatorum] $\dagger D U$

<34.> In CH2 e P1 il testo si interrompe qui, con la fine della serie di allegazioni: in CH2 manca infatti del tutto la solutio, mentre per quanto riguarda il ms. Paris, Bibliothèque Nationale, lat. 4601, essa non è riportata al fol. $5 r$ (P1), ma al fol. $26 r$ (P2). Nel ms. LE2 invece è presente solo la solutio, mentre le allegazioni sono riportate, in una tradizione diversa, da LE1: su quanto detto v. supra, parte III $\S 6$.

Eadem ... etc $\mathrm{om}$. $\mathrm{CA} 1 \mathrm{O} 2 \mathrm{Wl}$ Eadem etc. superveniens etc. $\mathrm{CH} 1 \quad$ vel ... debetur om. $B R U$ moneta] $\dagger$ $V A 2$ debetur om. $C H 3 D U M I M U 1 M Z T R W O$ etc] que tempore contractus erat et contra $O 1 W 2$ que tempore contractus fuit $\mathrm{P} 2$ etc. et econtra $\mathrm{CH} 3$ etc. et contra $\mathrm{MZ}$ etc. vel superveniens sive maior sive minor $L E 2$

<35.> Solutio om. CA1 Eadem mensura ... et eadem moneta] Eadem moneta ... et eadem mensura CH1 P2 WO debet ... moneta] et eadem moneta debet prestari AV CA2 DU MA MI MU1 P3 R SI TO TR VA1 VA2 W1 vel eadem moneta debet prestari $O 2$ vel moneta debet prestari $B O$ et in eadem moneta debet prestari $C A 1$ prestari] prestare $O 1$ et $o m$. $C H 3$ vel ... Statuta $o m$. BAM1 vel] et $M I$ alia] alias $P 3$ CA1 prestetur] prestaretur $C A 2 M U 1$ habeatur] habetur $M I M Z$ debetur $V A 1$ abeatur $A V$ ratio] ratione $D U$

<36.> Statuta ... futuris $o m$. $W 1$ Statuta] statuta enim $B R U W O$ facta $o m$. SI VAI contractibus] negotiis $O 1 W 2$ dant] donant $A V B O C A 1 M U 1 O 2$ donat $M A R$ SI TO W1 datur BAM1 donatio CA2 non $o m$. SI preteritis om. SI est VA1 ut om. CH1 C. om. VA1 constitutionibus] eum CH3 Leges] 1. Leges $B R U P 2 W O$

$<37 .>$ Nec] Non $B O \quad$ quod ] quiod $P 3 \quad$ ff. om. $B R U M Z \quad$ de legatis II] II de legatis $C H 3 \quad$ II] I $M U 1$ $\S$ ] que $B A M 1$ Imperator] Imperatorum $W O$ Inperator $D U$ ultimol penultimo $O 1 W 2 \quad \mathbf{d e}^{2}$ ] ff. de $B A M 1$ CH1 CH3 1.... tabernam om. BRE $\quad$ 1. om. BAM1 BAM2 BRU CH1 CH3 LE2 MI MZ O1 SI VA1 W2 WO e esp. 1. AV leg. con segno abbreviativo soprascritto LA Cum] con W1 tabernam] tabul. con segno 
testamenti vel contractus erant in hereditate, vel in bonis debitoris et in taberna, vel quin petatur utilitas, que erat tempore delicti: <39.> sed dicit, etiam in plus agi posse, vel dicitur, quod in primis duabus legibus facit illud tenor verborum testatoris; <40.> sicut et in ff. de conditionibus et demonstrationibus, Filie $\S$ ultimo (D. 35.1.28). <41.> Sic enim dixerat: quod supererit ex hereditate tempore mortis, vel, que in ea erit, cum moriar. $<42$. $>$ Item cum dicit tabernam, verisimile est id actum, ut res presentes et postea permutate vel substitute teneantur pignori, quia cotidie fit earum permutatio. $<43$. $>\mathrm{Nec}$ obstat, quod legitur in ff. de rebus creditis 1. Vinum (D. 12.1.22), quia ibi non loquitur in

abbreviativo sulla 1 . BRU OI taberna TO de $^{3}$ ] ff. de $B A M 1 C H 1 C H 3$ Infitiando] inficiendo $A V D U$ LA LE2 MA P2 P3 R VA1 VA2 WO infatiendo CA1 CA2 § om. LA quia BAM1

<38. Q Quia in] $\dagger C H 1$ his] hiis $D U L E 2 M U 1 T O T R V A 2 W O$ legibus] casibus $V A 2$ possint peti ea] peti possint ea $B R U$ possint] possit $B A M 1 C H 3 O 2 V A 1$ ea $O m$. $B R E C H 1$ testamenti vel contractus] contractus vel testamenti $L E 2 \quad$ vel $^{1}$ ] et $P 2$ erant] erat $C A 1 C A 2$ VA2 hereditate] hereditate erant $O 2$ in bonis] hominis $P 2$ imbonis $C A 2$ et om. DU TR in ${ }^{3}$ om. BAM1 CH1 CH3 MI MZ O1 O2 SI VA1 W1 $W 2 W O$ de $B R U$ quin om. WI quid $B A M 1$ cum $T R$ utilitas] hereditas $B R U$ que erat $o m$. $C H 1$ WO delicti] debiti $B R U$

<39.> dicit] dico $O 1 W 2$ dicitur quod $C H 1$ etiam in plus] in plus etiam $W O$ etiam] que $B R E$ in plus] amplius $B R U \quad$ in $^{1}$ om. $C H 1 O 2 P 2 W 1$ im $C A 1$ possel possit $C H 1$ dicitur om. TO in $\left.^{2}\right]$ im $C A 1$ primis om. $O 2 \quad$ legibus] legibus rep. $W 1 \quad$ facit] fecit $D U$ illud tenor] scilicet detior $B A M 1$ tenor] teneor $A V M A M Z P 2 P 3$ testatoris] testatorum $V A 2$

$<\mathbf{4 0}$.> sicut ... ultimo om. BAM2 sicut] sicuc $P 2$ et in om. MI P2 IIII TR et ${ }^{1}$ om. BAM1 BRE BRU $C H 3 D U L E 2 M Z R \quad$ in] illud $O 2 W 1 \mathrm{im} D U$ ff. om. $O 2$ de] de mo. con mo. esp. $M I$ conditionibus et demonstrationibus] conditione indebiti $B R E$ et demonstrationibus om. $L E 2$ Filie] in Filie $L E 2$

<41.> Sic] si O2 R W1 sicut BAM2 BRE CA1 CA2 CH1 CH3 DU MU1 enim om. BAM2 BRE CA1 CH3 $R$ erat $M U 1$ dixerat] dixerant $R$ dixit $L E 2$ quod] que $B R U$ supererit] supererant $A V L E 2 M U 1 P 2 P 3$ $R$ super erant $D U T R$ supererat $C A 2 C H 3 M Z$ supererunt $B R U$ super erunt $M A \quad$ ex om. BO CHI WO de $C H 3 W 2$ esset ex $W 1$ hereditate om. $C H 1 W O$ tempore] ex tempore $W 2$ que in] quin $B R E$ que] qui $R$ in ea] mea BAM1 BRU CA2 P2 TR W2 ea] eam VA2 erit] erunt AVBO BRUCA1 CA2 CH1 DU LA LE2 MA MU1 O1 O2 P3 R SI TR VA1 VA2 W1 W2 erat $P 2$ erant TO moriar] mortueiar con $\mathrm{t} u \mathrm{e}$ esp. VA2

$<\mathbf{4 2}$.> Item ... dicit] Item ut dicit cum $S I$ Item] Item ut $V A 1 \mathrm{ff}$. esp. Item $O 1$ cum dicit] condictionibus $P 2$ cum] animi $B A M 1$ dicit] om. $M U 1 \quad$ est $o m . O 2$ id] ad $A V$ ut res] ut res rep. $B O$ et $o m$. $D U$ $T R W 1$ posteal postea $\dagger L E 2$ permutate] mutate $C H 1 M I$ vel] vel et $M I$ et $C H 1$ P2 VAl WO teneantur] teneatur $B A M 1 O 2 V A 1$ teneant $P 2$ quia] qui $D U$ cotidie] $V A 1$ cotitidie con $\mathrm{i}^{1}$ esp. $V A 2$ tota die $C A 2$ earum] eorum $C H 1 P 2 W O$ permutatio] mutatio $C A 2$ 
alia mensura, sed in eadem. $<44 .>$ Queritur autem de extimatione rei, quo tempore sit spectanda. $<45$. $>$ Alie leges contrarie non videntur esse digne responsione.

$<$ 43.> Nec obstat] obest $P 2$ in ff. om. LE2 in ${ }^{1}$ ] esp. CH3 om. BAMI CHI DU MI MZ O1 O2 SI TR

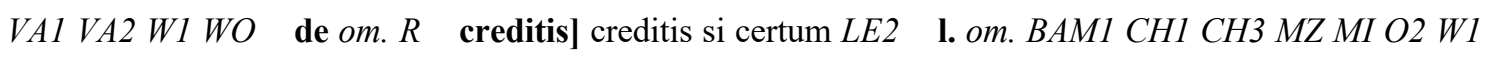
Vinum] annum $\mathrm{CH} 3$ non om. $O 2$ loquitur] loqui $O 2$ in alia] ita $B A M 1$ sed in eadem $\mathrm{om}$. $S I$ <44.> extimatione] existimatione $S I V A 1$ extraminactione $C H 3$ rei om. $C H 3$ quo] que $A V$ sit] sint $\mathrm{CH} 3$ spectanda] expectanda $\mathrm{CH} 3$ prestanda $\mathrm{CH} 1 \mathrm{MI}$ WO

<45.> Alie] alia $M Z$ leges] vel $C H 3$ videntur] videtur $C A 2 M Z P 2$ VA1 VA2 WI esse om. MI digne] digniores MU1 responsione] responsionis AV BO CA1 CA2 DU LA MA R TO TR VA1 respontsiones VA2 respossionis $P 3$ pensionis $M U 1$ 
2. Variazioni nell'ordine delle allegazioni

BRU: .. <3. $>,<16 .>,<4 .>, \ldots,<13 .>,<15 .>,<14 .>,<22 .>\ldots,<29 .>,<31 .>,<30 .>$, $<32$. $>,<32$. $>,<34$. $>$...

$\mathrm{CH} 1: \ldots<3 .>,<16 .>,<4 .>\ldots$

$\mathrm{CH} 2: \ldots,<28 .>,<30 .>,<29 .>,<31 .>\ldots$

CH3: .. $<31 .>,<33 .>,<32 .>\ldots$

MI: .. $<3 .>,<16 .>,<4 .>\ldots$

$\mathrm{MZ}: \ldots<7 .>,<16 .>,<8 .>, \ldots,<15 .>,<16 .>,<17 .>\ldots$

$\mathrm{O} 1: \ldots<3 .>,<15 .>,<5 .>,<8 .>,<4 .>,<7 .>,<14 .>,<9 .>,<10 .>,<11 .>,<22 .>, \ldots,<28 .>$, $<30 .>,<29$. $>,<32 .>,<31 .>,<33$. $>\ldots$

O2: .. $<28.1>,<30 .>,<29 .>,<31 .>\ldots$

$\mathrm{P} 1: \ldots<28 .>,<30 .>,<29 .>,<31 .>\ldots$

W1: .. $<28.1 .>,<30 .>,<29 .>,<31 .>\ldots$

W2: .. $<3 .>,<14 .>,<5 .>\ldots$

WO: $\ldots<3 .>,<16 .>,<4 .>\ldots,<13 .>,<15 .>,<14 .>, \ldots$ 